\title{
Estudio fenológico de angiospermas leñosas mediterráneas del Jardín Botánico de la Universidad de Málaga (España)
}

\author{
Marta Recio y Patricia Silva-Marín \\ Dpto. Biología Vegetal. Facultad de Ciencias. Universidad de Málaga. 29071-Málaga
}

\section{Correspondencia}

M. Recio

e-mail: martarc@uma.es

Recibido: 18 enero 2018

Aceptado: 3 octubre 2018

Publicado on-line: noviembre 2018

\section{Resumen}

Se ha realizado un estudio sobre el comportamiento fenológico de las angiospermas leñosas mediterráneas presentes en el Jardín Botánico de la Universidad de Málaga. Para cada especie se ha elaborado una tabla o calendario anual que incluye fenofases vegetativas y reproductivas. Se analiza y discute la distribución y duración de cada una de las fenofases estudiadas, así como su diversidad en el jardín. Se comparan todos los resultados, tanto de manera particular como general, con los publicados de áreas naturales. También se ha determinado el patrón de comportamiento fenofásico que presenta cada especie, habiendo resultado un total de siete patrones diferentes, la mayoría de tipo superpuesto (con crecimiento vegetativo y floración solapados en su ciclo anual) y minoritariamente de tipo secuencial. Se concluye que el crecimiento vegetativo ocurre fundamentalmente en dos periodos: primavera y otoñoinvierno; mientras que la formación de yemas florales así como la floración tiene lugar fundamentalmente durante la primavera, siendo marzo el mes que mayor número de especies la inician. Un porcentaje importante de especies presentan periodos largos (3 ó 4 estaciones del año) de crecimiento vegetativo $(30 \%)$, floración $(22 \%)$, fructificación (45\%) y dispersión (32\%). Se ha comprobado que la mayoría de las especies leñosas mediterráneas cultivadas en el jardín presentan periodos de crecimiento vegetativo y reproducción sexual que coinciden en duración, inicio y final con los publicados en medios naturales de la provincia de Málaga, por lo que los resultados fenológicos que se obtengan de los ejemplares cultivados en el jardín se pueden extrapolar a los que habitan en medios naturales.

Palabras clave: Fenología, angiospermas, fanerófitos, caméfitos, Mediterráneo, jardín botánico.
Abstract
Phenological study of mediterranean woody angiosperms in the Botanical Garden of the University of Malaga (Spain)
A study on the phenological behavior of Mediterranean woody angiosperms present in the Botanical Garden of the University of Malaga has been carried out. For each species an annual table or calendar that includes vegetative and reproductive phenophases has been prepared. The distribution and duration of each phenophases studied, as well as their diversity in the garden, are analyzed and discussed. All results are compared, both in a particular and general way, with those published from natural areas. The phenophasic behavior pattern of each species has also been determined, resulting in a total of seven different patterns, most of which are superimposed (with vegetative growth and flowering overlap in their annual cycle) and a minority of sequential type. It is concluded that the vegetative growth occurs mainly in two periods: spring and autumn- winter, while the formation of floral buds as well as flowering takes place mainly during the spring, with March being the month when the greatest number of species initiate it. A significant percentage of species have long periods (3 or 


\begin{abstract}
4 seasons) of vegetative growth (30\%), flowering (22\%), fruiting (45\%) and dispersion (32\%). It has been verified that the majority of Mediterranean woody species cultivated in the garden present periods of vegetative growth and sexual reproduction that coincide in duration, beginning and end with those published in natural environments of the province of Malaga. Therefore, the phenological results obtained from specimens grown in garden can be extrapolated to those of natural environments.
\end{abstract}

Key words: Phenology, angiosperm, woody species, Mediterranean, botanical garden

\section{Introducción}

La fenología es la ciencia que estudia los cambios estacionales en los órganos de los seres vivos durante su ciclo completo. Cuando se estudia los cambios estacionales que afectan a la estructura y forma de los seres vivos, se refiere entonces a la fenomorfología. Existen estudios fenomorfológicos que describen los cambios estacionales de órganos y morfología de las plantas durante su ciclo completo de vida en la región Mediterránea, entre los que podemos destacar los de Orshan $(1964,1989)$ Hoffmann (1972), Hoffmann \& Hoffmann (1976), Montenegro et al. (1979), Jow et al. (1980), Hoffmann \& Walker (1980), Aljaro \& Montenegro (1981), Westman (1981), Hoffmann (1981), Rutherford \& Panagos (1982), Montenegro (1987), Navarro \& Cabezudo (1998), Palacio \& Montserrat-Martí (2006), Navarro et al. (2009), Hidalgo-Triana et al. (2017). Estos estudios permiten detectar el origen, crecimiento, senescencia y desprendimiento de cada parte de una planta (hojas, ramas e inflorescencias) así como conocer mejor la estructura y el ciclo anual. Además, la relación de las fenofases de cada órgano con los cambios estacionales del medio nos permite obtener un mejor conocimiento de las relaciones planta-ambiente (Cabezudo et al., 1992). En el ámbito de los ecosistemas mediterráneos, se han desarrollado trabajos que aplican métodos fenomorfológicos que caracterizan a las formaciones vegetales, entre los que podemos destacar los de Orshan (1982, 1986,1989), PérezLatorre \& Cabezudo $(2002,2006)$, Pérez-Latorre et al. $(2007,2010)$.

Este trabajo se ha realizado solamente para angiospermas leñosas, es decir aquellas plantas con flores, semillas y frutos cuyos tallos, independientemente de su tamaño, han desarrollado estructuras de consistencia dura y rígida, árboles, arbustos y matas. Estas plantas se caracterizan porque son perennes, es decir, su ciclo biológico lo completa en más de un año, en contraposición a las plantas anuales o terófitos.
Además tienen las yemas de renovación por encima del nivel del suelo. Por tanto, este trabajo se aplica exclusivamente a fanerófitos y caméfitos. Por otro lado, este trabajo se realiza solamente para especies que se distribuyen en la región mediterránea, cuyo macroclima es mediterráneo, caracterizado por unos inviernos húmedos y templados, y unos veranos secos y calurosos.

Un jardín botánico tiene muchos recursos para realizar estudios científicos, entre los que se podrían incluir los estudios fenológicos. Además es un espacio que está bien cuidado y cuenta con una importante diversidad de especies botánicas, en su mayoría identificadas y etiquetadas con sus nombres científicos.

El Jardín Botánico de la Universidad de Málaga se construyó para cultivar plantas con fines experimentales, de estudio, de aclimatación, de divulgación, en definitiva crear un jardín con fines científicos que además pudiera cumplir con la finalidad ornamental de cualquier jardín (www.jardinbotanico.uma.es). Entre los trabajos científicos que se están llevando en el Jardín Botánico de la UMA, están los realizados por alumnos y profesores de la Facultad de Ciencias de la misma universidad. Uno de los temas de investigación ha sido el que se publica aquí, el estudio fenológico de angiospermas que habitan en la cuenca mediterránea, un lugar muy importante del planeta por ser un punto caliente de biodiversidad a nivel mundial (Médali \& Quétzel, 1999).

\section{Objetivos}

1. Seguimiento fenológico de diferentes fenofases (crecimiento vegetativo de dolicoblastos y braquiblastos, formación yemas florales, floración, fructificación, dispersión de diásporas y caída de hojas), mediante observación directa de los ejemplares de angiospermas leñosas de origen mediterráneo cultivadas en el Jardín Botánico de la Universidad de Málaga. 
2. Análisis particulares (para cada especie) y generales (para el conjunto de las especies examinadas) de las fenofases de crecimiento y reproducción (sexual), y determinación de patrones de comportamiento fenológico.

3. Estudio comparativo entre el comportamiento fenológico en el jardín y el que tiene en su medio natural, mediante consulta bibliográfica.

4. Análisis de la diversidad de comportamientos fenológicos obtenidos entre las especies estudiadas de angiospermas leñosas mediterráneas del jardín.

\section{Material y métodos}

\section{Área de estudio}

El Jardín Botánico de la Universidad de Málaga se inició en 1997 y se encuentra situado en el Campus de Teatinos, entre la facultad de Filosofía y Letras y la facultad de Ciencias. En una parcela rectangular, encerrado entre muros y rejas, se sitúan las colecciones que son pertenecientes a las Escuelas Botánicas, mientras que en los jardines de las distintas facultades y en el bulevar Louis Pasteur del Campus se encuentran otras colecciones temáticas como son el Hortus o el Arboretum. Las colecciones que pertenecen a las Escuela Botánica (http://www.jardinbotanico.uma. es/jardinbotanico/index.php/escuelas-botanicas) responden a una ordenación de las especies en función de su parentesco constituyendo con esto a una de las partes más importantes del Jardín Botánico. Está destinada a mostrar ordenadamente los diversos grupos sistemáticos que componen el mundo vegetal, estando adaptado al diagrama en círculo propuesto por Stebbins (1974). Esta parcela de la Escuela Botánica, comúnmente conocida como Jardín Botánico propiamente dicho, consta de más de 50 cuadrados de colecciones taxonómicas y más de 10 zonas con colecciones temáticas (http://www.jardinbotanico.uma.es/ jardinbotanico/index.php/colecciones-tematicas).

Se seleccionaron todos aquellas especies que fuesen de origen mediterráneo y cuyo biotipo fuera fanerófito o caméfito. Hay una importante representatividad de especies de plantas leñosas mediterráneas (30\%) en la parcela de la colección de flora árida-semiárida. No obstante el conjunto de las especies mediterráneas está representado y repartido por casi todas las partes del Jardín Botánico debido a la diversidad sistemática y filogenética de dicho conjunto de especies (Tabla 1). Las especies estudiadas pertenecen a 27 familias distintas, siendo Rosáceas y Fagáceas las familias más representadas (ambas con un valor porcentual de 10\%), seguido de Lamiáceas (8\%), Leguminosas, Oleáceas y Chenopodiáceas

\begin{tabular}{llll}
\hline Familia & $\%$ & Familia & $\%$ \\
\hline Adoxaceae & 2 & Lauraceae & 2 \\
Anacardiaceae & 4 & Leguminosae & 6 \\
Apocynaceae & 2 & Malvaceae & 2 \\
Arecaceae & 2 & Moraceae & 2 \\
Asclepiadaceae & 2 & Myrtaceae & 2 \\
Asparagaceae & 2 & Oleaceae & 6 \\
Buxaceae & 4 & Plumbaginaceae & 2 \\
Cistaceae & 2 & Rhamnaceae & 4 \\
Celastraceae & 2 & Rosaceae & 10 \\
Chenopodiaceae & 6 & Solanaceae & 4 \\
Ericaceae & 2 & Tamaricaceae & 4 \\
Fabaceae & 4 & Ulmaceae & 2 \\
Fagaceae & 10 & Verbenaceae & 2 \\
Lamiaceae & 8 & & \\
\hline
\end{tabular}

Tabla 1. Diversidad de familias de angiospermas leñosas mediterráneas estudiadas (porcentaje en número de especies).

Table 1. Families diversity of Mediterranean woody angiosperms studied (percentage in number of species).

(con un 6\% de representatividad cada una de ellas). Respecto a los biotipos, la mayoría son árboles (macrofanerófitos, 48\%) y arbustos (nanofanerófitos, 42\%), siendo minoritario las matas leñosas (caméfitos, 10\%).

Algunas especies, como Pistacia terebinthus, Cistus albidus y Lavandula dentata, desaparecieron en el Jardín Botánico por lo que se estudiaron las que estaban cultivadas en el Hortus, un pequeño jardín situado entre la facultad de Filosofía y Letras y el muro oriental del Jardín Botánico. En el caso de Myrtus comunis, se estudió un ejemplar con menos caracteres hibridados (hojas, flores y frutos de tamaño más parecido al de los ejemplares naturales) situado en el parterre más meridonal de los jardines de la facultad de Ciencias.

En el caso de especies dioicas, se ha observado que en el Jardín Botánico solamente se encuentra cultivado un ejemplar de un sexo (masculino) de algarrobo (Ceratonia siliqua). Por ello, se ha estudiado la fenología de un ejemplar de otro sexo (femenino) cultivado en los jardines de la facultad de Ciencias. En estos casos, los resultados fenológicos presentados corresponderían a la especie y no al ejemplar unisexual.

\section{Estudio fenológico}

El estudio fenológico se realizó mediante observación directa de los ejemplares cultivados en el jardín. Se presenta, para cada especie, 


\begin{tabular}{|c|c|c|c|}
\hline Taxon & $\begin{array}{l}\mathrm{N}^{\circ} \text { herbario (fecha de } \\
\text { recolección) }\end{array}$ & Taxon & $\begin{array}{l}\mathrm{N}^{\circ} \text { herbario (fecha de } \\
\text { recolección) }\end{array}$ \\
\hline Anabasis articulata & MGC79855 (24/10/2014) & Quercus coccifera & MGC80579 (31/03/2015) \\
\hline Arbutus unedo & MGC80515 (18/11/2014) & Quercus faginea & MGC11151 (12/03/2015) \\
\hline Asparagus albus & MGC79861 (23/10/2014) & Quercus rotundifolia & $\begin{array}{l}\text { MGC80510 (26/11/2014) } \\
\text { MGC80646 (12/03/2015) }\end{array}$ \\
\hline Atriplex halimus & MGC79853 (24/10/2014) & Quercus suber & MGC80511 (26/11/2014) \\
\hline Buxus balearica & MGC80516 (18/11/2014) & Rosmarinus officinalis & MGC79851 (24/10/2014) \\
\hline Buxus sempervirens & MGC10797 (26/02/2015) & Salsola papillosa & MGC79854 (24/10/2014) \\
\hline Ceratonia siliqua & $\begin{array}{l}\text { MGC79858 }(24 / 10 / 2014) \\
\text { MGC79862 }(11 / 11 / 2014) \\
\text { MGC80513 }(13 / 11 / 2014)\end{array}$ & Spartium junceum & MGC10651 (21/04/2015) \\
\hline Cercis siliquastrum & $\begin{array}{l}\text { MGC11149 (12/03/2015) } \\
\text { MGC80577 (01/04/2015) }\end{array}$ & Tamarix africana & MGC10589 (25/03/2015) \\
\hline Cotoneaster granatensis & MGC79860 (23/10/2014) & Tamarix parviflora & MGC80578 (31/03/2015) \\
\hline Crataegus monogyna & $\begin{array}{l}\text { MGC10679 (21/04/2015) } \\
\text { MGC11156 (12/03/2015) }\end{array}$ & Thymus hyemalis & $\begin{array}{l}\text { MGC80514 (13/11/2014) } \\
\text { MGC80581 (01/04/2015) }\end{array}$ \\
\hline Maytenus senegalensis & MGC79850 (23/10/2014) & Thymus mastichina & MGC80512 (26/11/2014) \\
\hline Myrtus communis & MGC11155 (12/03/2015) & Ulmus minor & $\begin{array}{l}\text { MGC11150 (12/03/2015) } \\
\text { MGC80583 (01/04/2015) }\end{array}$ \\
\hline Phillyrea angustifolia & MGC10803 (04/03/2015) & Viburnum tinus & MGC11148 (12/03/2015) \\
\hline Pistacia lentiscus & MGC80580 (01/04/2015) & Withania frutescens & MGC79859 (23/10/2014) \\
\hline Prunus spinosa & MGC79856 (24/10/2014) & Ziziphus lotus & MGC79857 (23/10/2014) \\
\hline Pyrus bourgueana & MGC79690 (26/11/2014) & & \\
\hline
\end{tabular}

Tabla 2. Testigos del material estudiado depositado en el Herbario MGC.

Table 2. Studied plant sheets stored in the MGC Herbarium.

una tabla o calendario fenológico, siguiendo la metodología y el modelo propuesto por Orshan (1989). Este método ha sido bien aceptado por la comunidad científica relacionada con este campo de investigación, sobre todo en ecosistemas mediterráneos, siendo numerosos los trabajos publicados que siguen su metodología.

Esta tabla fenológica consta de 12 columnas que corresponden a cada uno de los meses del año, razón por la que se le ha denominado calendario fenológico. Hay que tener en cuenta que, aunque comienza en enero, el ciclo anual de las plantas vasculares normalmente comienza en octubre, coincidiendo con el comienzo del otoño. Las filas de la tabla o calendario fenológico corresponden a las distintas fases vegetativas y reproductivas definidas por Orshan (1989).

Las fases fenológicas vegetativas estudiadas y definidas por Orshan (1989) son el crecimiento vegetativo de dolicoblastos (CVD) y braquiblastos (CVB), así como la caída de hojas de ambos tipos de ramas (CHD y $\mathrm{CHB})$. El crecimiento vegetativo consiste en un incremento de la longitud de las ramas y/o aparición de nuevas hojas visibles a simple vista. El crecimiento vegetativo de dolicoblastos está generalmente relacionado con la elongación de ramas, siendo allí donde nacen los nuevos braquiblastos y donde continúa con la aparición de nuevas hojas. El término caída de hojas se aplica a la muerte de hojas, tanto si se han desprendido como si siguen unidas al vástago, por lo que se refiere a la presencia de hojas muertas intactas.

Las fases fenológicas reproductivas estudiadas y definidas por Orshan (1989) son: formación de yemas florales (PF), floración (FL), fructificación (FR) y dispersión (DS). Una yema es un brote embrionario constituido por hojas o por esbozos foliares del que se desarrollarán flores, ramas u hojas (Azcón \& Talón, 2008). Una yema floral es cada uno de los brotes de la planta que al desarrollarse forman las inflorescencias o flores individuales. La fenofase de formación de yemas florales consiste, evidentemente, en la aparición de estos brotes diferenciados, visibles a simple vista. La fenofase de floración consiste en el 
desarrollo de las flores, desde la apertura de las más tempranas hasta la marchitez de las tardías de la misma temporada. La fenofase de fructificación es el ovario maduro y que contiene las semillas, además de cualquier parte adyacente que puede fusionarse con él en la madurez. La dispersión es el trasporte de las diásporas que contienen la semilla.

Se ha incluido una última fila que correspondería a la observación de yemas indiferenciadas $(Y)$, ya que es un dato importante. Estas yemas tienen el inconveniente de que no se pueden asociar a una $u$ otra fase (vegetativa o reproductiva) ya que hasta que no se diferencian no se sabe cuál será su papel biológico.

El seguimiento fenológico se ha llevado a cabo desde julio de 2014 hasta junio de 2015, realizándose observaciones y anotaciones en la hoja de tablas fenológicas desde una vez al mes como mínimo hasta una vez a la semana. Hay una tabla fenológica para cada especie y las observaciones se han realizado siempre en el mismo ejemplar, siendo un solo individuo el seleccionado y estudiado, excepto en el caso de especies dioicas como se ha comentado anteriormente.

Muchas de estas fenofases se encuentran testificadas mediante pliegos de herbario que se han preparado para tal efecto, encontrándose depositados en el Herbario MGC (Tabla 2).

\section{Determinación del patrón fenológico}

Se ha determinado también el patrón fenológico de cada especie, siguiendo el método de Montenegro et al. (1989), Pérez-Latorre \& Cabezudo (2002) y Pérez-Latorre et al. (2010). Estos patrones se definen en función del comienzo, final y coincidencia (o no) del crecimiento y floración durante el ciclo anual. A cada uno de los modelos o patrones de comportamiento fenológico se le asigna una letra. Los patrones A, B, C, D y E fueron propuestos y descritos por Montenegro et al. (1989), mientras que el patrón $F$ fue propuesto y descrito por Pérez-Latorre \& Cabezudo (2002), y el último (G) por Pérez-Latorre et al. (2010).

A: Desarrollo de yemas florales durante el periodo de crecimiento vegetativo. La floración ocurre solamente cuando se ha completado el crecimiento de las ramas y la expansión de las hojas.

B: Similar al patrón A, pero las yemas florales se desarrollan al final del periodo de crecimiento vegetativo. Es un patrón fenofásico totalmente secuencial.

C: El desarrollo de las yemas florales y la floración ocurre dentro del periodo de crecimiento vegetativo, terminando ambos al mismo tiempo. Es un patrón de solapamiento fenofásico.

D: La floración tiene lugar antes del crecimiento vegetativo. Es un patrón totalmente secuencial.

$\mathrm{E}$ : Difiere del anterior en que las yemas florales se desarrollan antes del comienzo del crecimiento vegetativo, mientras que la floración sucede dentro del crecimiento vegetativo. Tiene una parte secuencial y otra parte solapada.

F: Patrón que difiere del anterior en que está totalmente solapado. Difiere del patrón $\mathrm{C}$ en que la floración termina antes que termine el crecimiento vegetativo.

G: La formación de yemas florales (prefloración) y floración coincide con el crecimiento vegetativo, pero el crecimiento se paraliza en un momento determinado mientras la floración continúa, y después continúa creciendo finalizándose la fase de crecimiento después de que finalice la de floración.

\section{Resultados y discusión}

Los resultados fenológicos obtenidos para cada especie son los que se presentan en la Figura 1 y se resumen en la Tabla 3.

En general, se ha obervado que para la gran mayoría de las especies leñosas mediterráneas examinadas coinciden la duración, inicio y final de la floración con la publicada en la obra Flora Vascular de Andalucía Oriental, el alcornocal de Bohornoque y algunas comunidades de bosque, arbusteda y matorral serial de Málaga, incluso con un jardín de Italia. No obstante, algunas especies leñosas inician su floración antes en el jardín botánico estudiado. Eso último puede explicarse por la situación del jardín, próximo a la costa y a baja altitud, lo que hace las temperaturas sean más cálidas que en otros espacios naturales donde habitan. Algunas especies leñosas mediterráneas, como p. ej. Quercus rotundifolia, presentan una gran amplitud ecológica para el factor clima, habitando en los pisos termoclimáticos termo-, meso- y supramediterráneo, cada una con rangos de temperatura distinto. De la misma manera, el hecho de que la floración sea muchas veces más corta en el jardín estudiado que en el espacio natural malagueño, posiblemente se deba a la misma razón explicada anteriormente, al hecho de ser especies ampliamente distribuidas desde el punto de vista termoclimático, siendo por tanto más duradera la floración para la especie, pero no para el individuo.

\section{Crecimiento vegetativo}

Se presenta la distribución mensual (Fig. 2) 
Anabasis articulata

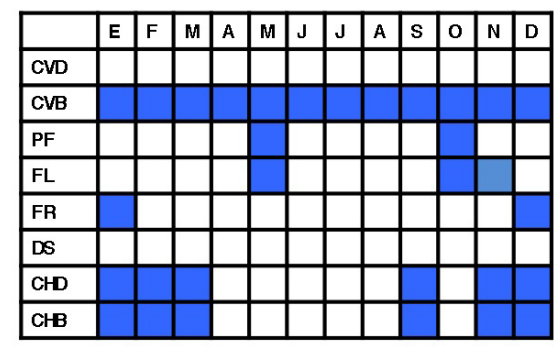

Atriplex halimus

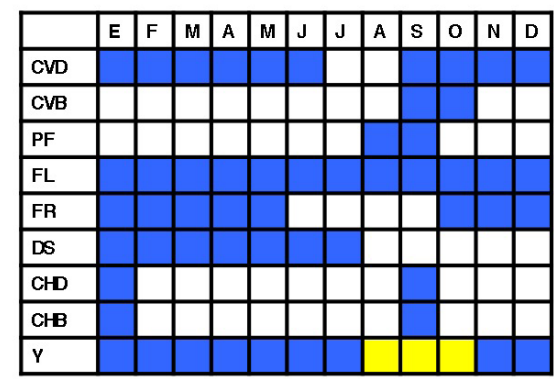

Ceratonia siliqua (masculino)

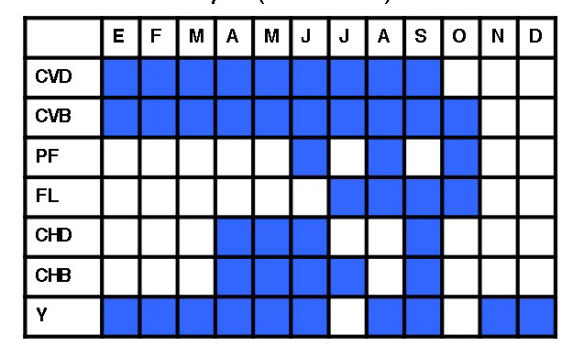

Chamaerops humilis

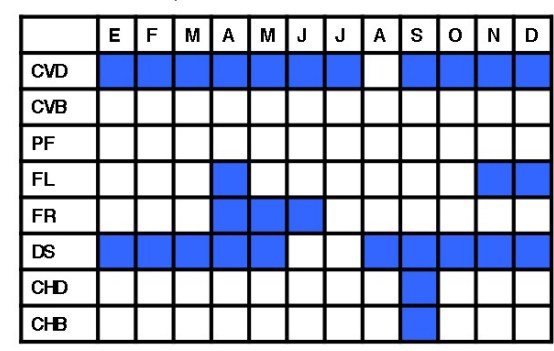

Crataegus monogyna

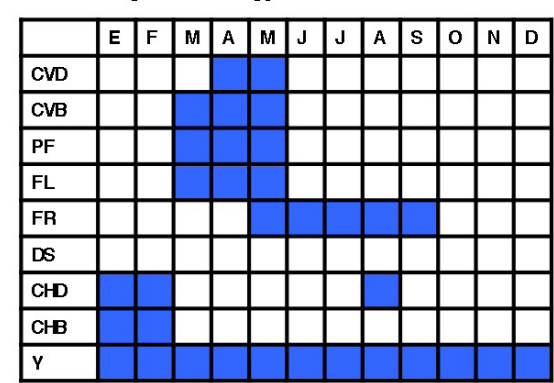

Arbutus unedo

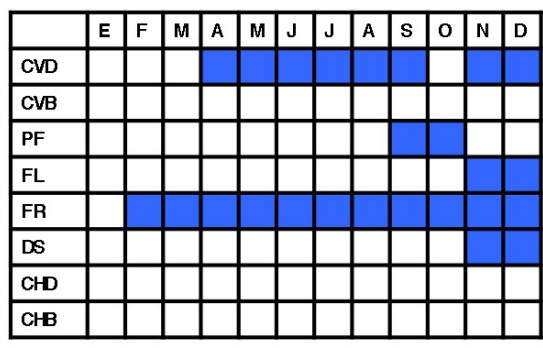

Buxus balearica

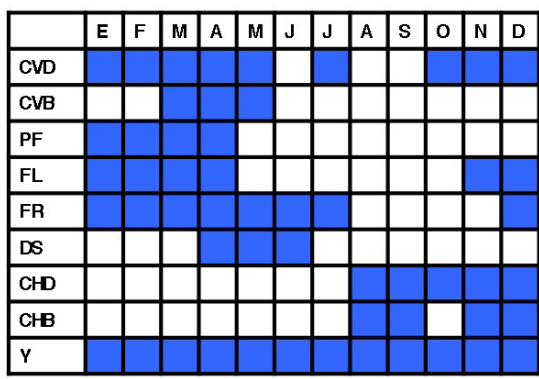

\section{Ceratonia siliqua (femenino)}

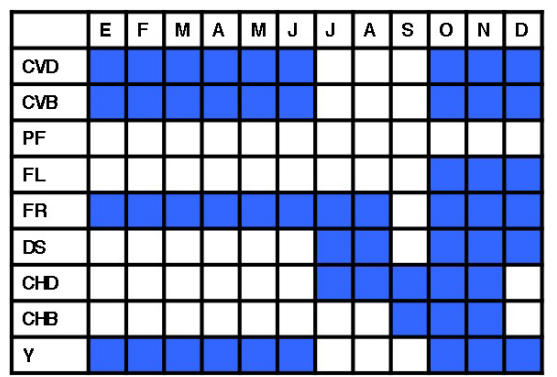

Cistus albidus

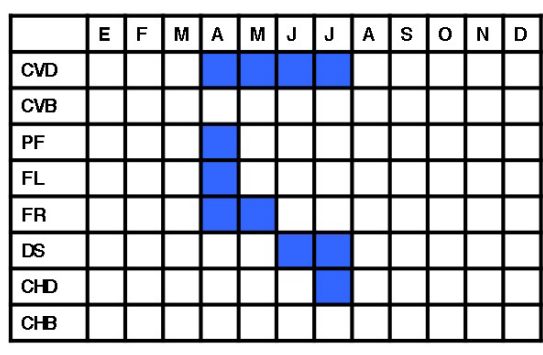

Lavandula dentata

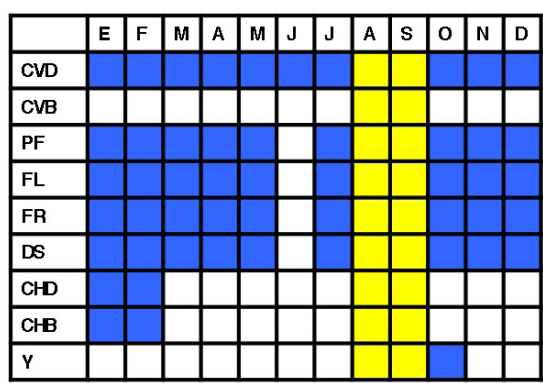

Asparagus albus

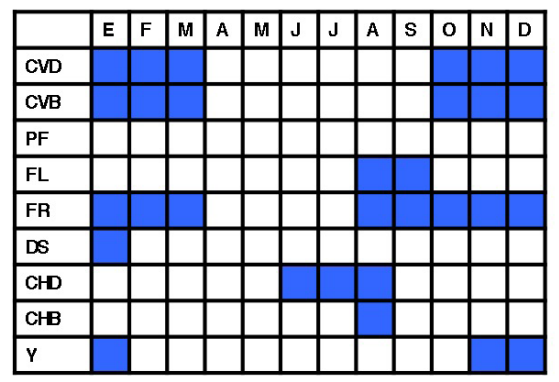

Buxus sempenirens

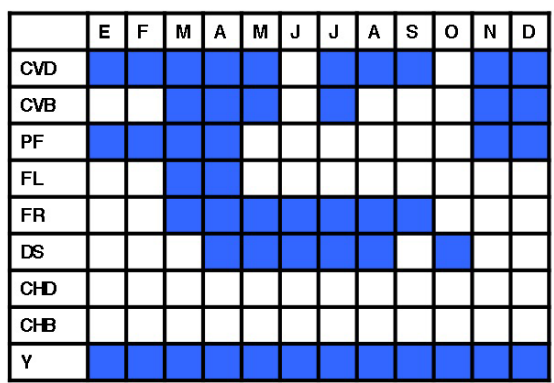

Cercis siliquastrum

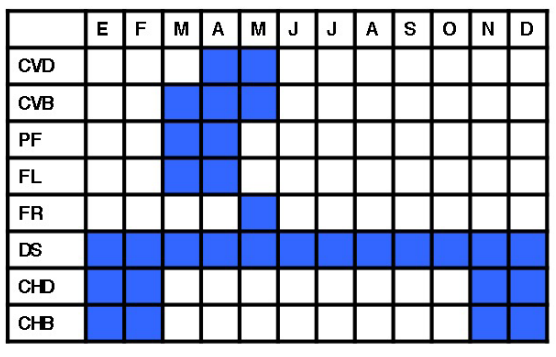

Cotoneastergranatensis

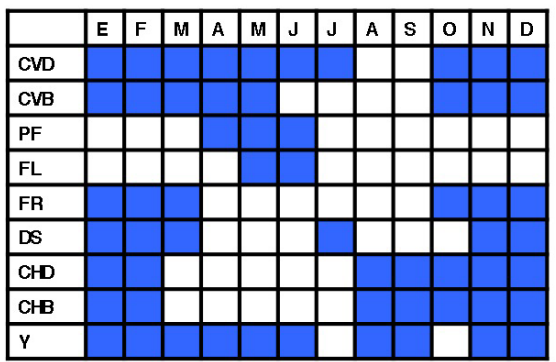

Limoniastrum monopetalum

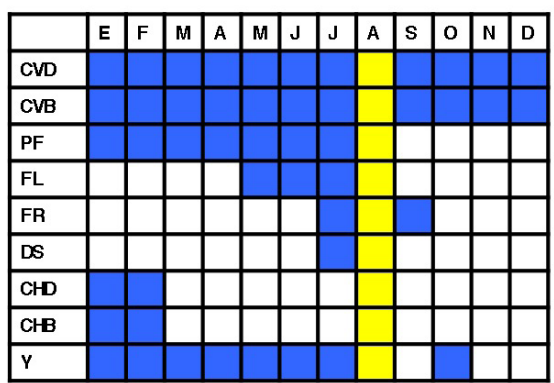

Figura 1. Diagrama fenológico anual de las especies estudiadas.

Figure 1. Annual phenological diagram of the species studied. 

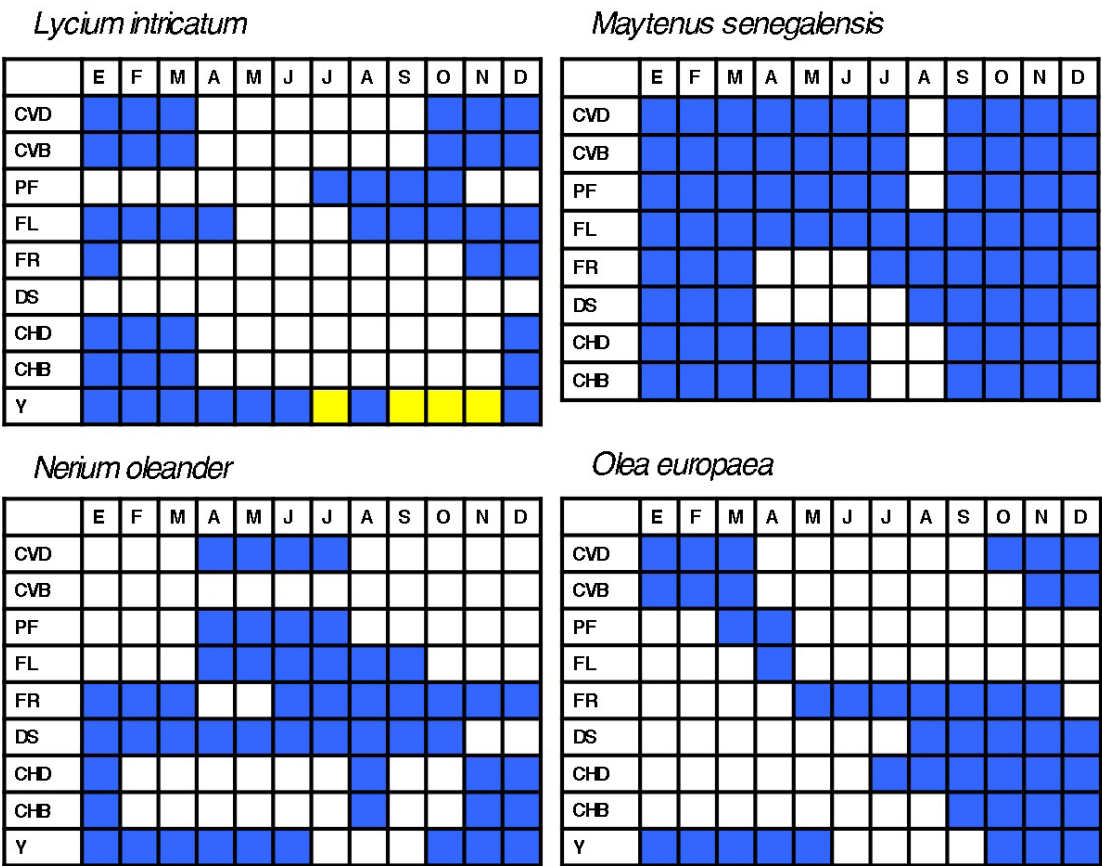

\section{Phillyrea angustfolia}

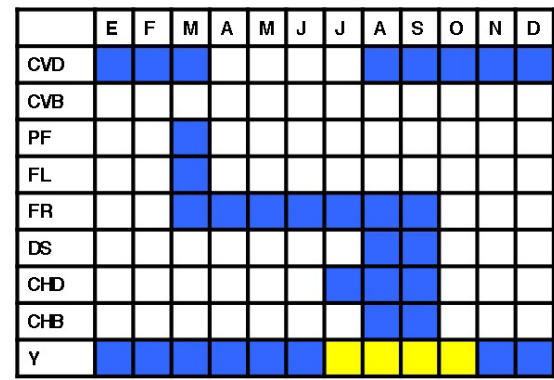

Prunus spinosa

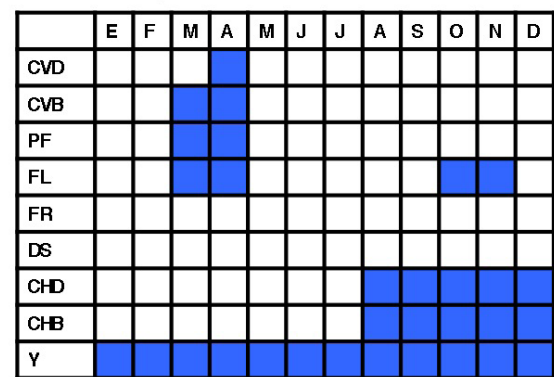

Quercus faginea

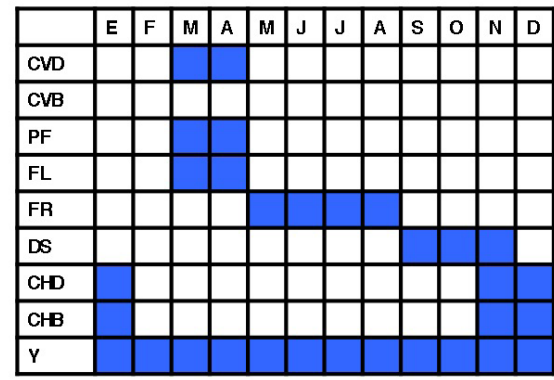

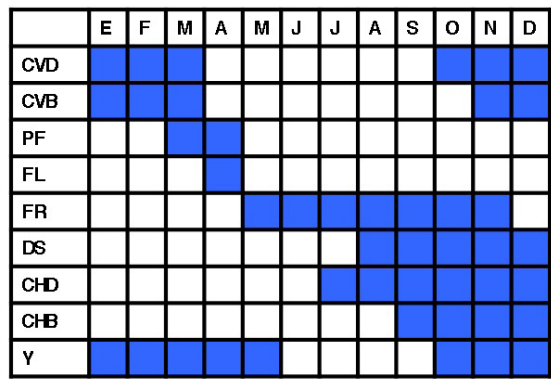

\section{Pistacia lentiscus}

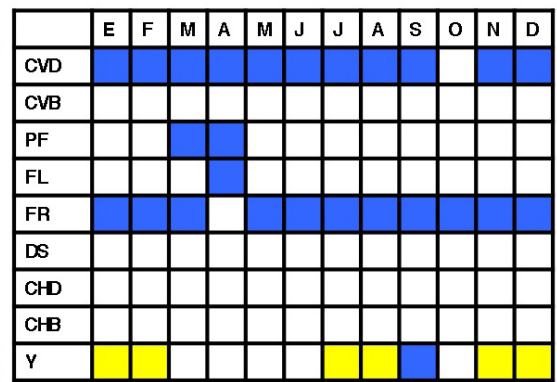

Pynus bourgaeana

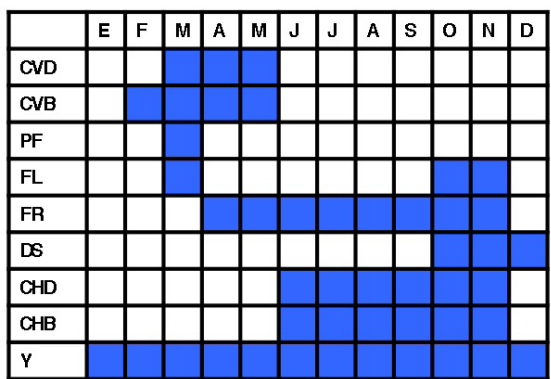

\section{Quercus rotundifolia}

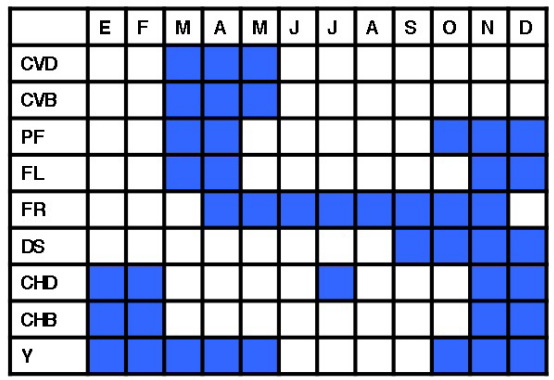

\section{Olea europaea}

Myrtus communis

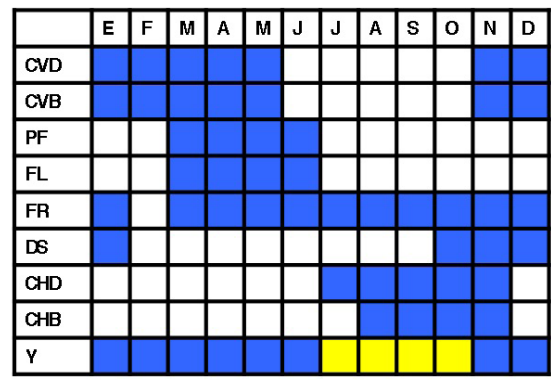

Peniploca angustifolia

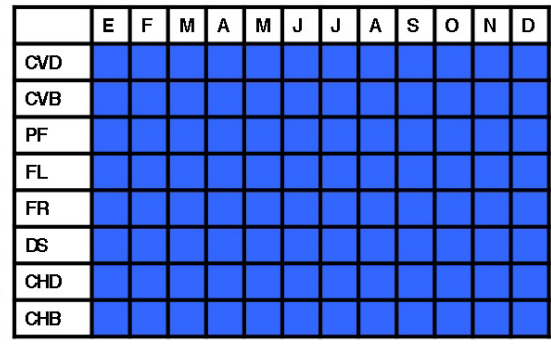

Prunus mahaleb

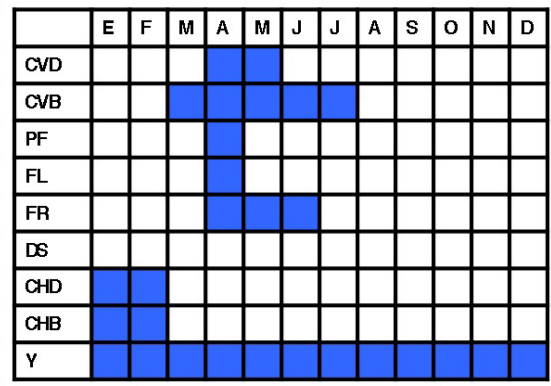

Quercus coccifera

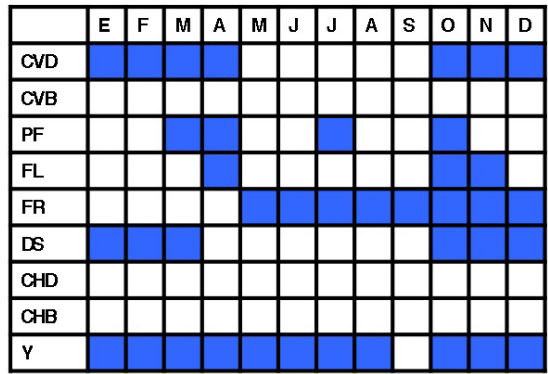

Quercus suber

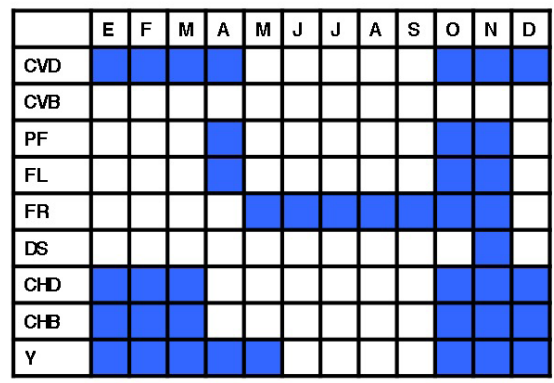

Figura 1 (Continuación). 
Retama sphaerocapa

\begin{tabular}{|l|l|l|l|l|l|l|l|l|l|l|l|l|}
\hline & E & F & M & A & M & J & J & A & S & O & N & D \\
\hline CVD & & & & & & & & & & & & \\
\hline CVB & & & & & & & & & & & & \\
\hline PF & & & & & & & & & & & & \\
\hline FL & & & & & & & & & & & & \\
\hline FR & & & & & & & & & & & & \\
\hline DS & & & & & & & & & & & & \\
\hline CHD & & & & & & & & & & & & \\
\hline CHB & & & & & & & & & & & & \\
\hline Y & & & & & & & & & & & & \\
\hline
\end{tabular}

Salsola papillosa

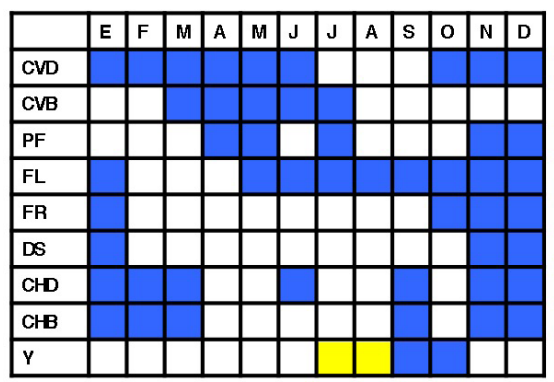

Tamanx paniflora

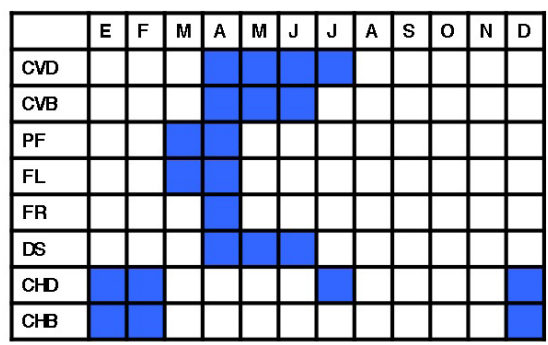

Vibumum tinus

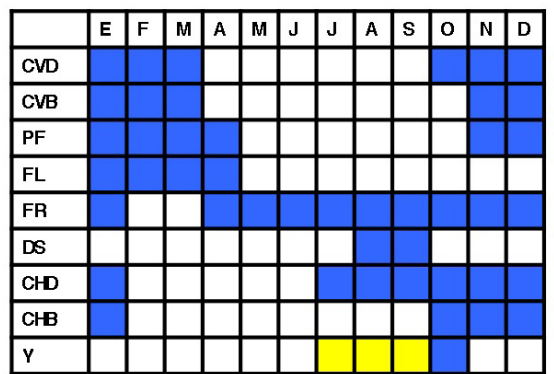

\section{Zziphus btus}

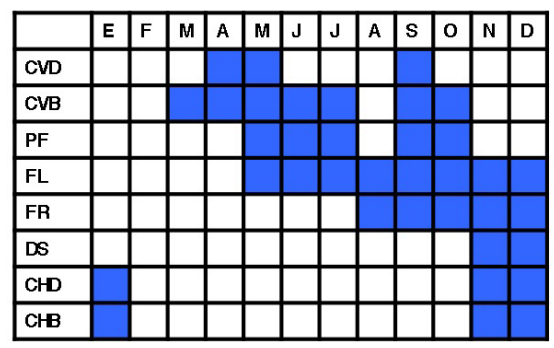

Rhamnus hycioides

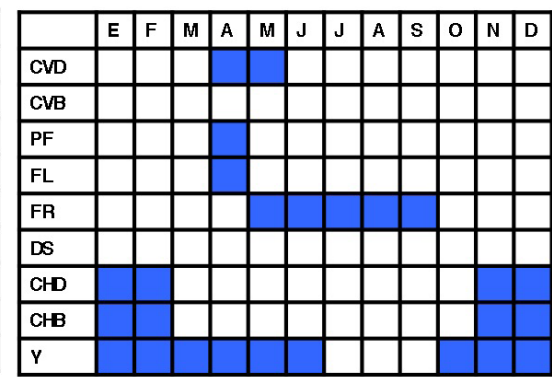

Spartium junceum

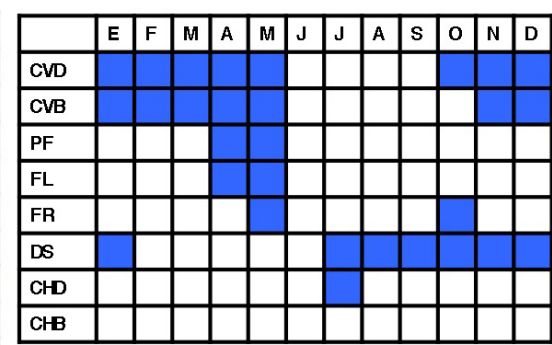

Thymus mastichina

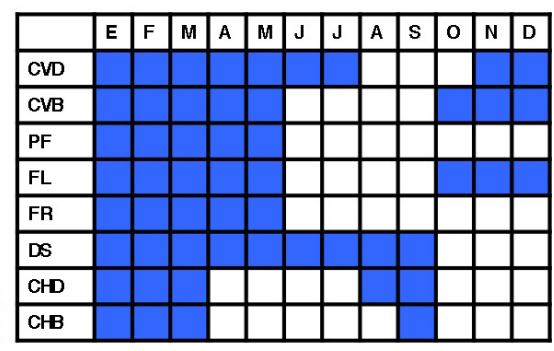

Vitex agnus-castus

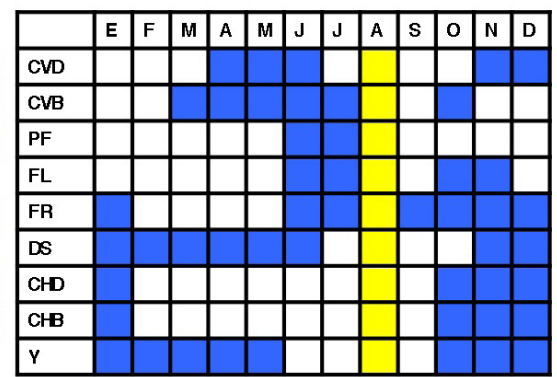

Rosmarinus officinalis

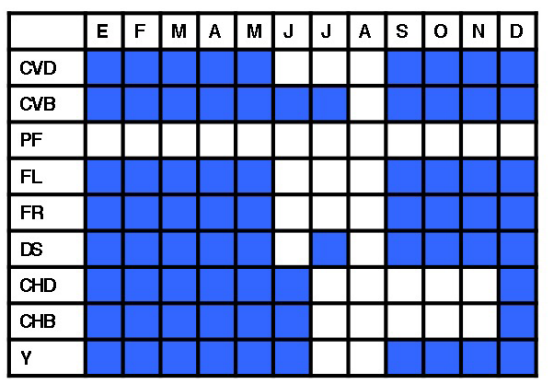

Tamarix africana

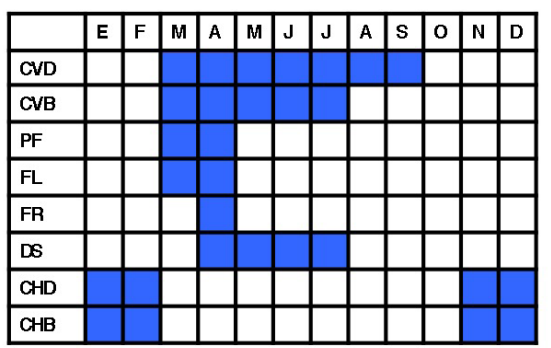

Ulmus minor

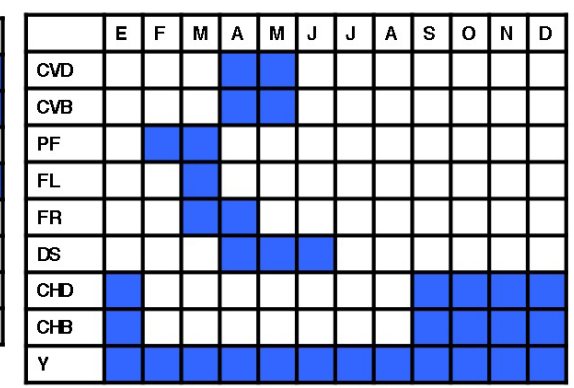

Withania frutescens

\begin{tabular}{|c|c|c|c|c|c|c|c|}
\hline & \begin{tabular}{|l|}
$E$ \\
\end{tabular} & 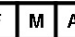 & \begin{tabular}{l|l|l}
$A$ & $M$
\end{tabular} & \begin{tabular}{l|l}
$J$ \\
\end{tabular} & \begin{tabular}{|l|l}
$\mathrm{A}$ & \\
\end{tabular} & \begin{tabular}{l|l|l}
$s$ & 0
\end{tabular} & \begin{tabular}{l|l}
$\mathrm{N}$ & $\mathrm{D}$ \\
\end{tabular} \\
\hline CVD & & & & & & & \\
\hline CVB & & & & & & & \\
\hline $\mathrm{PF}$ & & & & & & & \\
\hline$F L$ & & & & & & & \\
\hline$F R$ & & & & & & & \\
\hline DS & & & & & & & \\
\hline CHD & & & & & & & \\
\hline $\mathrm{CHB}$ & & & & & & & \\
\hline$y$ & & & & & & & \\
\hline
\end{tabular}

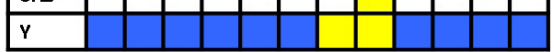


y estacional (Tabla 4) del crecimiento vegetativo en número de especies. Se observa que existen dos periodos de crecimiento más abundante entre las especies estudiadas: primavera en mayor representación, seguido de otoño-invierno. En verano el crecimiento vegetativo se da en muy pocas especies (Retama sphaerocarpa, Tamarix africana, Tamarix parviflora, Vitex agnus-castus y Ziziphus lotus). El 24\% de las especies examinadas crece vegetativamente solo en primavera, mientras que un $10 \%$ lo hace en otoño-invierno y otro $10 \%$ en primavera-verano. Es de destacar que el $56 \%$ de las especies examinadas presenta crecimiento vegetativo durante 3 ó 4 estaciones del año, exceptuando siempre en uno, dos o tres meses de verano.

Apenas existen diferencias entre la distribución mensual del crecimiento vegetativo de dolicoblastos y el de braquiblastos.

Estos resultados difieren de los examinados en el ecosistema mediterráneo de Chile, donde primavera e invierno son las estaciones con mayor número de especies en crecimiento vegetativo y donde en otoño no hubo ninguna especie en fase de crecimiento (Montenegro et al., 1989). Tampoco coincide con los analizados en Francia (Floret et al., 1989), Israel (Orshan, 1989) y Sudáfrica (Le Roux et al., 1989).

\section{Floración}

Se presenta la distribución mensual (Fig. 3) y estacional (Tabla 4) de la floración en número de especies. En general podemos afirmar que la fenofase de formación de yemas florales de la mayoría de especies leñosas mediterráneas cultivadas en el jardín tiene lugar generalmente en primavera, siendo agosto el mes con menor número de especies en esta fenofase, y marzo el mes en que mayor número de especies la inician (Fig. 3).

$Y$, respecto a la fenofase de floración, se observa que existen dos periodos más abundantes entre las especies analizadas: finales de inviernoinicios de primavera (marzo y abril), en mayor representación, seguido de otoño (octubre, noviembre y diciembre). Por otro lado, el $45 \%$ de las especies examinadas florecen unicamente en primavera (Tabla 3). En primavera más otoño sólo lo hacen un $7 \%$. Es de destacar que un $22 \%$ de las especies examinadas florecen durante 3 ó 4 estaciones del año. Estos resultados difieren de los encontrados en Francia (Floret et al., 1989), donde más del $70 \%$ de las especies florece en primavera (fundamentalmente en mayo) y el $19 \%$ de las especies florece en verano. Además ninguna especie florece durante las cuatro estaciones del año y sólo un $1,4 \%$ de las especies lo hace en tres estaciones.

Durante el verano y el invierno la floración se da en menor número de especies leñosas mediterráneas estudiadas. Asparagus albus y el ejemplar femenino de Ceratonia siliqua solamente florece durante los meses de verano. También florecen en verano, pero tras haberse iniciado en primavera, Nerium oleander y Retama sphaerocarpa y Vitex agnus-castus (este último continuando en otoño). Con floración invernal solamente se han encontrado dos especies: Viburnum tinus y Withania frutescens. Hay tres especies (Anabasis articulata, Prunus spinosa y Pyrus bourgaeana) que florecen en dos estaciones (primavera y otoño) separadas por el verano.

\section{Fructificación y dispersión}

En la Fig. 3 también se presenta la distribución mensual de las fases de fructificación y dispersión de la diáspora, no habiéndose observado variación significativa a lo largo del ciclo anual. En todos los meses del año existe entre un 15\% y $25 \%$ de especies en fase de fructificación y entre un $12 \%$ y $20 \%$ de especies en fase de dispersión. Por tanto, respecto a la fructificación y dispersión no ha habido una mayoría de especies que lo hagan en una estación determinada del año.

El $30 \%$ de las especies examinadas fructifican durante las tres estaciones del año, y el 15\% durante las cuatro estaciones. Únicamente fructifica en primavera un $18 \%$ (Tabla 4). Hay que tener encuenta que hay una alta diversidad de tipos de frutos entre las especies leñosas mediterráneas cultivadas en el jardín botánico, y que la mayoría son secos (88\%), ya que constituyen una adaptación al clima.

Respecto a la dispersión, el $20 \%$ de las especies estudiadas la realizan durante tres estaciones del año y el $11 \%$ durante todo el ciclo anual (Tabla 3).

En general, podemos concluir que la fenofase de fructificación y dispersión es larga para las especies leñosas mediterráneas.

\section{Caída de hojas}

Existen dos tipos de especies leñosas en cuanto a este carácter o fenofase: los que tienen caída total de hojas en la estación desfavorable (generalmente invierno) y los que son perennifolias. Éstas últimas siempre presentan hojas pero se van renovando y suelen presentar una caída parcial de hojas en la estación desfavorable (invierno o más comúnmente verano en el caso de plantas mediterráneas). En nuestro jardín solamente hay 11 especies caducifolias de distribución 
mediterránea. Existen además tres especies leñosas mediterráneas que son áfilas (sin hojas ni filoclados).

En la Fig. 4 se representa la distribucción mensual de la caída de hojas de dolicoblastos y braquiblastos en número de especies examinadas, observándose un mayor número de especies que pierden sus hojas durante finales de otoño y principios del invierno $(22 \%$ de las especies examinadas, según Tabla 3). Otro periodo en el que pierden hojas un porcentaje representativo de especies es el verano (18\%) y el verano-otoño (18\%). Un $20 \%$ de las especies pierden sus hojas en dos periodos desfavorables separados (invierno y verano). Prácticamente no hay especies que pierdan su hojas durante la primavera, solamente lo hacen Maytenus senegalensis, Periploca angustifolia y Rosmarinus officinalis. Curiosamente en estas tres especies arbustivas perennifolias superponen las fenofases de crecimiento $y$ fructificación durante la primavera, estación en la que para ahorrar energía pierden parcialmente sus hojas.

Apenas existe diferencias significativas entre la distribución mensual de la la caída de hojas de distinto origen (dolicoblasto o braquiblasto).

En general podemos concluir que la fenofase de caída de hojas para la gran mayoría de las especies estudiadas ocurre durante el verano, sobre todo a finales, pero también hay una buena representatividad durante el otoño y principios de invierno, siendo muy escasas las especies que lo hacen durante primavera.

\section{Patrones fenofásicos}

El patrón mayoritario ha resultado ser en $\mathrm{C}$
(58\% de las especies analizadas). Le sigue el patrón $E(17 \%)$. Del resto de los patrones hay una representación minoritaria pero al menos existen de una a tres especies de cada uno de los diferentes patrones fenológicos. Por tanto, entre las especies leñosas mediterráneas cultivadas en el jardín botánico de la UMA, están representados siete patrones fenofásicos distintos (Fig. 5).

Según Pérez-Latorre \& Cabezudo (2002) se pueden agrupar dos tipos básicos de patrones: $A+B+D$, en el que se empieza el crecimiento antes que la floración y las fenofases no son coincidentes; y $\mathrm{C}+\mathrm{E}+\mathrm{F}$, en el que crecimiento y floración están solapados. El primer grupo de patrones fenofásicos lo presenta el $13 \%$ de las especies examinadas, mientras que el segundo grupo lo constituye un $87 \%$ de las especies examinadas. Parece ser que la razón por la que la mayoría de las especies examinadas presentaran patrones fenofásicos solapados sea el estrés hídrico estival carcaterístico del clima mediterráneo.

Patrones secuenciales de tipo $A$ han sido determinados para Arbutus unedo, Phillyrea angustifolia y Vitex agnus-castus, de tipo B para Olea europaea, y de tipo D para Asparagus albus y Ulmus minor. La determinación del patrón $\mathrm{D}$ para Asparagus albus había sido realizada por PérezLatorre et al. (2010) en comunidades arbustivas edafoxerófilas del litoral oriental de la provincia de Málaga.

El patrón G lo presentan muy pocas especies, habiéndose determinado solamente en Maytenus senegalensis subsp. europaeus, Atriplex halimus y Salsola papillosa, todas ellas presentes en comunidades arbustivas edafoxerófilas de la costa oriental semiárida-árida de Málaga y Andalucía

Tabla 3. Resumen de los resultados fenológicos obtenidos para cada especie estudiada en el Jardín Botánico de la Universidad de Málaga. Coincide con lo publicado en Flora Vascular de Andalucía Oriental (Blanca et al. 2011) (1). Coincide con lo publicado en Flora Vascular de Andalucía Occidental (Valdés et al. 1987) (2). Coincide el trabajo publicado por Pérez-Latorre \& Cabezudo (2002) en comunidades naturales malagueñas de bosque y matorral serial (3). Coincide el trabajo publicado por Pérez-Latorre et al. (1996) en un alcornocal de Boornoque (Sierra de las Nieves, Málaga) (4). Coincide el trabajo publicado por Pérez-Latorre et al. (2010) en una comunidad edafoxerófila de la costa oriental mañagueña (5) Coincide el trabajo publicado por Hidalgo \& Cabezudo (1994) en un matorral de la Sierra de Mijas (6). Coincide el trabajo publicado por Cabezudo et al. (1992) en un alcornocal con encinas de los Montes de Málaga (7). Coincide el trabajo publicado por Spano et al. (1999) en un jardín de Italia (8). Jardín Botánico (JB). Dolicoblasto (D). Braquiblasto (B).

Table 3. Summary of the phenological results obtained for each species studied in the Botanical Garden of the University of Málaga. Same with the published in Flora Vascular de Andalucía Occidental (Valdés et al. 1987) (2). Same with the published by Pérez-Latorre \& Cabezudo (2002) in natural communities of forest and scrub serial from Malaga (3 Same with the published by Pérez-Latorre et al. (1996) in a cork oak from Boornoque (Sierra de las Nieves, Málaga) (4). Same with the published by Pérez-Latorre et al. (2010) in a edaphoxerophile community from east coast of Malaga (5) Same with the published by Hidalgo \& Cabezudo (1994) in a cork oak from Sierra de Mijas (6). Same with the published by Cabezudo et al. (1992) in a cork oak from Montes de Málaga (7). Same with the published by Spano et al. (1999) in a garden from Italy (8). Botanical Garden (JB). Dolichoblast (D). Brachyblast (B). 


\begin{tabular}{|c|c|c|c|c|c|}
\hline Especie & Patrón & Crecimiento & Reproducción & Floración & $\begin{array}{l}\text { Referencias coincidentes. } \\
\text { Diferencias observadas }\end{array}$ \\
\hline Anabasis articulata & C & $\begin{array}{l}\text { Largo.Todo el año, } \\
\text { excepto Junio }\end{array}$ & Fenofases secuenciales & $\begin{array}{l}\text { Corta. } \\
\text { Doble: primav. y } \\
\text { otoño }\end{array}$ & 1 \\
\hline Arbutus unedo & $\mathrm{B}$ & $\begin{array}{l}\text { Primavera-verano } \\
\text { y otoño }\end{array}$ & Fenofases secuenciales & $\begin{array}{l}\text { Corta. } \\
\text { Otoño }\end{array}$ & $\begin{array}{l}1,3,4 . \text { En JB retrasa el } \\
\text { comienzo de la floración } \\
\text { un mes }\end{array}$ \\
\hline Asparagus albus & $\mathrm{D}$ & Otoño-invierno & Fenofases secuenciales & Corta.Verano & $1,2,5$ \\
\hline Atriplex halimus & $\mathrm{G}$ & $\begin{array}{l}\text { Largo.Excepto } \\
\text { Julio y Agosto }\end{array}$ & $\begin{array}{l}\text { Fenofases solapadas, } \\
\text { secuencial el inicio }\end{array}$ & $\begin{array}{l}\text { Larga. } \\
\text { Todo el año }\end{array}$ & $\begin{array}{l}\text { 1. Florece todo el año en } \\
\text { el JB }\end{array}$ \\
\hline Buxus balearica & C & $\begin{array}{l}\text { Largo D } \\
\text { Corto B (primav.) }\end{array}$ & Fenofases solapadas & $\begin{array}{l}\text { Invierno-principios } \\
\text { primav. }\end{array}$ & 1 \\
\hline Buxus sempervirens & C & $\begin{array}{l}\text { Largo D } \\
\text { Corto y doble B } \\
\text { (primav. y otoño) }\end{array}$ & Fenofases solapadas & $\begin{array}{l}\text { Corta. } \\
\text { Primavera }\end{array}$ & 1 \\
\hline Ceratonia siliqua & C & $\begin{array}{l}\text { Largo.Todo el año, } \\
\text { excepto otoño }\end{array}$ & $\begin{array}{l}\text { Fenofases solapadas, } \\
\text { secuencial el inicio }\end{array}$ & $\begin{array}{l}\text { Verano y principios } \\
\text { otoño. }\end{array}$ & $\begin{array}{l}1,2,6 . \text { En JB inicia un } \\
\text { mes antes las masculinas }\end{array}$ \\
\hline Cercis siliquastrum & $E$ & $\begin{array}{l}\text { Corto. } \\
\text { Primavera }\end{array}$ & $\begin{array}{l}\text { Fenofases solapadas, } \\
\text { excepto fructificación }\end{array}$ & $\begin{array}{l}\text { Corta. Primavera } \\
\text { ( } 2 \text { meses) }\end{array}$ & $\begin{array}{l}1,8, \text { pero en el } \\
\text { JB se adelanta un mes }\end{array}$ \\
\hline Chamaerops humilis & $\mathrm{F}$ & $\begin{array}{l}\text { Largo.Todo el año, } \\
\text { excepto Agosto }\end{array}$ & $\begin{array}{l}\text { Fenofases solapadas, } \\
\text { secuencial el inicio }\end{array}$ & $\begin{array}{l}\text { Corta. } \\
\text { Primavera }\end{array}$ & $1,2,5$ \\
\hline Cistus albidus & C & $\begin{array}{l}\text { Corto. } \\
\text { Primavera }\end{array}$ & $\begin{array}{l}\text { Fenofases solapadas de } \\
\text { yemas y floración, } \\
\text { secuencial el inicio de } \\
\text { fructificación y dispersión }\end{array}$ & $\begin{array}{l}\text { Corta. } \\
1 \text { mes (Abril) }\end{array}$ & 1,7 \\
\hline $\begin{array}{l}\text { Cotoneaster grana- } \\
\text { tensis }\end{array}$ & $\mathrm{C}$ & $\begin{array}{l}\text { Largo.Todo el año, } \\
\text { excepto verano }\end{array}$ & $\begin{array}{l}\text { Fenofases solapadas, } \\
\text { secuencial el inicio }\end{array}$ & $\begin{array}{l}\text { Corta. } \\
\text { Finales primavera } \\
\text { ( } 2 \text { meses) }\end{array}$ & 1 \\
\hline Crataegus monogyna & $\mathrm{F}$ & $\begin{array}{l}\text { Corto.Principios } \\
\text { primavera }\end{array}$ & $\begin{array}{l}\text { Fenofases solapadas, } \\
\text { excepto fructificación }\end{array}$ & $\begin{array}{l}\text { Corta. } \\
\text { Primavera }\end{array}$ & 1 \\
\hline Lavandula dentata & C & $\begin{array}{l}\text { Largo. } \\
\text { Todo el año }\end{array}$ & Fenofases solapadas & $\begin{array}{l}\text { Larga.Todo el año, } \\
\text { excepto Junio }\end{array}$ & $\begin{array}{l}\text { En el JB se adelanta } 2 \\
\text { meses respecto } \\
\text { a lo publicado en } 1\end{array}$ \\
\hline $\begin{array}{l}\text { Limoniastrum } \\
\text { monopetalum }\end{array}$ & C & $\begin{array}{l}\text { Largo.Todo el año, } \\
\text { excepto Septiem. }\end{array}$ & $\begin{array}{l}\text { Fenofases solapadas, } \\
\text { secuencial el inicio }\end{array}$ & $\begin{array}{l}\text { Primavera y } \\
\text { verano }\end{array}$ & 1 \\
\hline Lycium intricatum & $E$ & Otoño e invierno & Fenofases secuenciales & $\begin{array}{l}\text { Larga. Verano, } \\
\text { otoño e invierno }\end{array}$ & $\begin{array}{l}\text { En el JB no florece en } \\
\text { primavera }\end{array}$ \\
\hline $\begin{array}{l}\text { Maytenus } \\
\text { senegalensis subsp. } \\
\text { europaeus }\end{array}$ & $\mathrm{G}$ & $\begin{array}{l}\text { Largo. } \\
\text { Todo el año, } \\
\text { excepto Agosto }\end{array}$ & $\begin{array}{l}\text { Fenofases solapadas, } \\
\text { secuencial el inicio }\end{array}$ & $\begin{array}{l}\text { Larga. } \\
\text { Todo el año }\end{array}$ & $\begin{array}{l}\text { 1, pero en el JB también } \\
\text { florece en invierno } \\
\text { y primavera. } \\
5 \text {, pero en el JB inicia el } \\
\text { crecimiento antes }\end{array}$ \\
\hline Myrtus communis & C & $\begin{array}{l}\text { Largo. } \\
\text { Otoño-invierno y } \\
\text { primavera }\end{array}$ & Fenofases solapadas & $\begin{array}{l}\text { Invierno-primav. (4 } \\
\text { meses) }\end{array}$ & $\begin{array}{l}1,6,8, \text { pero en el JB inicia } \\
\text { la floración un mes antes }\end{array}$ \\
\hline Nerium oleander & $\mathrm{C}$ & $\begin{array}{l}\text { Corto. } \\
\text { Primavera }\end{array}$ & Fenofases solapadas & $\begin{array}{l}\text { Corta. } \\
\text { Primavera }\end{array}$ & 1 \\
\hline Olea europaea & B & Otoño e invierno & Fenofases secuenciales & Corta.1 mes (Abril) & 1 \\
\hline Periploca angustifolia & $\mathrm{F}$ & $\begin{array}{l}\text { Largo. } \\
\text { Todo el año }\end{array}$ & Fenofases solapadas & $\begin{array}{l}\text { Larga. } \\
\text { Todo el año }\end{array}$ & $\begin{array}{l}\text { 1, pero en el JB florece } \\
\text { todo el año }\end{array}$ \\
\hline Phillyrea angustifolia & A & $\begin{array}{l}\text { Largo. } \\
\text { Verano, otoño e } \\
\text { invierno }\end{array}$ & Fenofases solapadas & $\begin{array}{l}\text { Corta. } \\
1 \text { mes (Marzo) }\end{array}$ & $\begin{array}{l}3,4,6 \text {, coincide con el JB } \\
\text { en floración pero no en } \\
\text { crecimiento }\end{array}$ \\
\hline Pistacia lentiscus & $\mathrm{C}$ & $\begin{array}{l}\text { Largo.Todo el año, } \\
\text { excepto Octubre }\end{array}$ & $\begin{array}{l}\text { Fenofases solapadas, } \\
\text { secuencial el inicio }\end{array}$ & $\begin{array}{l}\text { Corta. } \\
1 \text { mes (Abril) }\end{array}$ & $\begin{array}{l}1,6,8 \text {, pero inicia un mes } \\
\text { antes que } 3 \text { y } 4\end{array}$ \\
\hline Prunus mahaleb & $E$ & $\begin{array}{l}\text { Corto. } \\
\text { Primavera }\end{array}$ & Fenofases solapadas & $\begin{array}{l}\text { Corta. } 1 \text { mes } \\
\text { (Abril) }\end{array}$ & 1 \\
\hline Prunus spinosa & $E$ & $\begin{array}{l}\text { Corto.Principios } \\
\text { primavera }\end{array}$ & Fenofases solapadas & $\begin{array}{l}\text { Corta. Doble: } \\
\text { primav. y otoño }\end{array}$ & 1 \\
\hline
\end{tabular}




\begin{tabular}{|c|c|c|c|c|c|}
\hline Especie & Patrón & Crecimiento & Reproducción & Floración & $\begin{array}{l}\text { Referencias coincidentes. } \\
\text { Diferencias observadas }\end{array}$ \\
\hline Pyrus bourgueana & $\mathrm{E}$ & Corto. Primavera & Fenofases solapadas & $\begin{array}{l}\text { Corta.Doble: } \\
\text { primav. y otoño }\end{array}$ & 1 \\
\hline Quercus coccifera & C & $\begin{array}{l}\text { Largo. } \\
\text { Otoño-invierno y } \\
\text { principios primav. }\end{array}$ & Fenofases solapadas & $\begin{array}{l}\text { Doble: principios } \\
\text { primav. y otoño }\end{array}$ & $\begin{array}{l}\text { 1, pero en el JB florece } \\
\text { en Abril }\end{array}$ \\
\hline Quercus faginea & $\mathrm{E}$ & $\begin{array}{l}\text { Corto. } \\
\text { Primavera }\end{array}$ & $\begin{array}{l}\text { Fenofases solapadas de } \\
\text { yemas y floración, } \\
\text { secuencial la } \\
\text { fructificación y dispersión }\end{array}$ & $\begin{array}{l}\text { Doble: primav. y } \\
\text { otoño }\end{array}$ & $\begin{array}{l}1,2,4, \text { pero en el JB inicia } \\
\text { la floración un mes antes }\end{array}$ \\
\hline Quercus rotundifolia & $\mathrm{C}$ & $\begin{array}{l}\text { Corto. } \\
\text { Primavera }\end{array}$ & $\begin{array}{l}\text { Fenofases solapadas de } \\
\text { yemas y floración, } \\
\text { secuencial la } \\
\text { fructificación y dispersión }\end{array}$ & $\begin{array}{l}\text { Doble: primav. y } \\
\text { otoño }\end{array}$ & $\begin{array}{l}\text { 1, pero en el JB es más } \\
\text { corta e intensa en } \\
\text { primavera, y en otoño es } \\
\text { más leve }\end{array}$ \\
\hline Quercus suber & $\mathrm{C}$ & Otoño e invierno & $\begin{array}{l}\text { Fenofases solapadas de } \\
\text { yemas y floración, } \\
\text { secuencial la } \\
\text { fructificación y } \\
\text { dispersión }\end{array}$ & $\begin{array}{l}\text { Corta. } \\
1 \text { mes (Abril) }\end{array}$ & $\begin{array}{l}\text { 1, pero en el JB sólo flore- } \\
\text { ce en Abril } \\
3,4 \text {, pero en el JB } \\
\text { la floración se adelanta un } \\
\text { mes, y no hay crecimiento } \\
\text { en primavera }\end{array}$ \\
\hline Retama sphaerocarpa & $\mathrm{C}$ & Primavera y verano & $\begin{array}{l}\text { Fenofases solapadas, } \\
\text { secuencial el inicio de } \\
\text { fructificación }\end{array}$ & Primav. y verano & $\begin{array}{l}\text { No coincide con } 3 \text {, ni en } \\
\text { crecimiento ni en } \\
\text { reproducción, posiblemen- } \\
\text { te debido al estrés ya que } \\
\text { está cultivada } \\
\text { erróneamente en el JB en } \\
\text { suelo arenoso } \\
\text { (parcela de psammófilas) }\end{array}$ \\
\hline Rhamnus lycioides & C & $\begin{array}{l}\text { Largo.Todo el año, } \\
\text { excepto verano }\end{array}$ & $\begin{array}{l}\text { Fenofases solapadas, } \\
\text { secuencial el inicio }\end{array}$ & $\begin{array}{l}\text { Corta. } \\
\text { Primavera }\end{array}$ & $\begin{array}{l}\text { 1, pero en el JB la flora- } \\
\text { ción es más corta }\end{array}$ \\
\hline Rosmarinus officinalis & C & $\begin{array}{l}\text { Largo.Todo el año, } \\
\text { excepto Agosto }\end{array}$ & Fenofases solapadas & $\begin{array}{l}\text { Larga.Todo el año, } \\
\text { excepto verano }\end{array}$ & 1,6 \\
\hline Salsola papillosa & $\mathrm{G}$ & $\begin{array}{l}\text { Largo. } \\
\text { Desde Octubre, } \\
\text { excepto verano }\end{array}$ & Fenofases secuenciales & $\begin{array}{l}\text { Larga. } \\
\text { Primavera, verano } \\
\text { y otoño }\end{array}$ & $\begin{array}{l}\text { 1, pero en el JB inicia la } \\
\text { floración un mes antes }\end{array}$ \\
\hline Spartium junceum & C & $\begin{array}{l}\text { Largo.Todo el año, } \\
\text { excepto verano }\end{array}$ & Fenofases solapadas & $\begin{array}{l}\text { Corta. } \\
\text { Primav. ( } 2 \text { meses) }\end{array}$ & $1,2,8$ \\
\hline Tamarix africana & $\mathrm{F}$ & Primav. y verano & Fenofases solapadas & $\begin{array}{l}\text { Corta.Principios de } \\
\text { pimav. ( } 2 \text { meses) }\end{array}$ & 1 \\
\hline Tamarix parviflora & $E$ & Primav. y verano & Fenofases solapadas & $\begin{array}{l}\text { Corta.Principios de } \\
\text { pimav. ( } 2 \text { meses) }\end{array}$ & $\begin{array}{l}\text { 1, pero en el JB la flora- } \\
\text { ción es más temprana }\end{array}$ \\
\hline Thymus mastichina & C & $\begin{array}{l}\text { Largo.Todo el año, } \\
\text { excepto verano }\end{array}$ & Fenofases solapadas & $\begin{array}{l}\text { Larga. } \\
\text { Otoño, invierno y } \\
\text { primavera }\end{array}$ & $\begin{array}{l}1,6, \text { pero en el JB } \\
\text { la floración es más larga }\end{array}$ \\
\hline Ulmus minor & $\mathrm{D}$ & $\begin{array}{l}\text { Corto. } \\
\text { Primav. (2 meses) }\end{array}$ & Fenofases secuenciales & $\begin{array}{l}\text { Corta. } \\
\text { Principios de pi- } \\
\text { mav. (Marzo) }\end{array}$ & 1 \\
\hline Viburnum tinus & C & Otoño e invierno & $\begin{array}{l}\text { Fenofases solapadas de } \\
\text { yemas y floración, } \\
\text { secuencial el inicio de } \\
\text { fructificación y dispersión }\end{array}$ & $\begin{array}{l}\text { Invierno e inicio de } \\
\text { primavera }\end{array}$ & $\begin{array}{l}3 \text {, coincide con el JB en } \\
\text { reproducción, pero no en } \\
\text { crecimiento. } \\
\text { En el JB la floración es } 2 \\
\text { meses más adelantada, y } \\
\text { la fructifiación y caída de } \\
\text { hojas } 2 \\
\text { meses más prolongada }\end{array}$ \\
\hline Vitex agnus-castus & $A$ & Primav. y verano & Fenofases solapadas & $\begin{array}{l}\text { Verano y principio } \\
\text { de otoño }\end{array}$ & 1 \\
\hline Whitania frutescens & $\mathrm{F}$ & $\begin{array}{l}\text { Largo.Todo el año, } \\
\text { excepto verano }\end{array}$ & & $\begin{array}{l}\text { Larga. Finales de } \\
\text { verano, otoño e } \\
\text { invierno }\end{array}$ & 1,5 \\
\hline Ziziphus lotus & $A$ & Primav. y verano & Fenofases secuenciales & Primav. y verano & $\begin{array}{l}\text { 1, pero en el JB la flora- } \\
\text { ción se prolonga hasta fin } \\
\text { de año }\end{array}$ \\
\hline
\end{tabular}

Tabla 3. (Continuación). 
en general. Este patrón $G$ había sido propuesto por Pérez-Latorre et al. (2010) para Maytenus senegalensis.

En general podemos afirmar, tal como habían hecho Pérez-Latorre \& Cabezudo (2002), que el hecho heberse observado en este estudio una diversidad de comportamientos fenológicos en las especies leñosas medietrráneas cultivadas en el jardín sea consecuencia de la alta diversidad característica del Mediterráneo, tanto en especies como en comunidades y paisajes vegetales. Además, los comportamientos fenológicos difieren según sean una especie característica de una comunidad climácica (generalmente bosque) o de una etapa serial (arbusteda o matorral). Los distintos comportamientos fenofásicos son consecuencia de su adaptación al medio, por lo que estos tipos de estudios dan información muy útil para estudiar cambios globales como consecuencia del cambio climático.

\section{Conclusiones}

La gran mayoría de las especies leñosas mediterráneas estudiadas fenológicamente en el jardín presentan periodos de crecimiento vegetativo y reprodución (sexual) que coinciden en duración, inicio y final con los publicados en medios naturales de la provincia de Málaga. No obstante muchas especies presentan un inicio de floración más temprano y una duración más corta de la misma.

El crecimiento vegetativo de las especies leñosas mediterráneas cultivadas en el jardín ocurre fundamentalmente en dos periodos del año: primavera (en mayor representación, siendo marzo el mes en que mayor número de especies la inician) y otoño-invierno. En verano el crecimiento vegetativo se da en muy pocas especies. Por otro lado, aproximadamente un $30 \%$ de las especies examinadas fenológicamente crece durante todo o casi todo el ciclo anual.

La formación de yemas florales así como la floración de la mayoría de especies leñosas mediterráneas cultivadas en el jardín tiene lugar en primavera, siendo marzo el mes en que mayor número de especies la inician. Un $22 \%$ de las especies examinadas florecen durante 3 ó 4 estaciones del año.

Las fenofases de fructificación y dispersión son en general largas para las especies leñosas mediterráneas. Durante 3 ó 4 estaciones del año fructifican el $45 \%$ de las especies examinadas fenológicamente y el 32\% dispersan la diáspora.

El hecho de que se haya registrado un porcentaje importante de especies que cuyas fenofases de crecimiento vegetativo, floración, frucificación y dispersión se realiza durante periodos largos (3 ó 4 estaciones del año) parece indicar un cierto grado de independencia del clima en buena parte de las especies leñosas mediterráneas cultivadas en el jardín, pues normalmente las fases

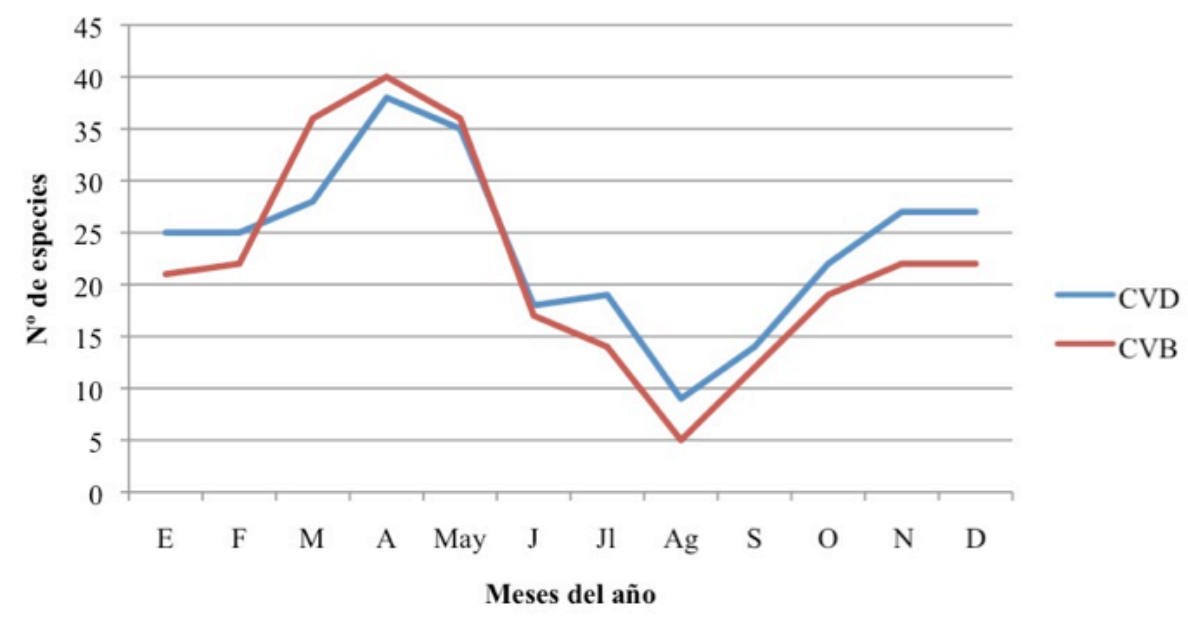

Figura 2. Número de especies leñosas mediterráneas que muestran fenofases de crecimiento vegetativo de dolicoblastos (CVD) y crecimiento vegetativo de braquiblastos (CVB) a lo largo de los meses del periodo estudiado (Julio 2014 - Junio 2015) en el Jardín Botánico de la Universidad de Málaga.

Figure 2. Number of Mediterranean woody species that show phenofases of dolichoblasts (CVD) and brachyblasts (CVB) vegetative growth throughout the months of the period studied (July 2014 - June 2015) in the Botanical Garden of the University of Malaga. 


\begin{tabular}{|l|l|l|l|l|l|l|l|l|l|l|l|}
\hline \multicolumn{1}{|c|}{ Estación } & \multicolumn{2}{c|}{ CV } & \multicolumn{2}{c|}{ FL } & \multicolumn{3}{c|}{ FR } & \multicolumn{3}{c|}{ DS } & \multicolumn{2}{c|}{ CH } \\
\hline & $\mathbf{n}$ & $\%$ & $\mathbf{n}$ & $\%$ & $\mathbf{n}$ & $\%$ & $\mathbf{n}$ & $\%$ & $\mathbf{n}$ & $\%$ \\
\hline Otoño & - & - & 1 & 2 & - & - & 6 & 17 & - & - \\
\hline Otoño + invierno & 5 & 10 & 2 & 5 & 3 & 8 & 5 & 15 & 8 & 22 \\
\hline Invierno & - & - & - & - & 1 & 2 & - & - & 2 & 5 \\
\hline Invierno + primavera & - & - & 2 & 5 & 2 & 5 & - & - & 1 & 3 \\
\hline Primavera & 11 & 24 & 19 & 45 & 7 & 18 & 3 & 9 & - & - \\
\hline Primavera + verano & 5 & 10 & 3 & 7 & 6 & 15 & 2 & 6 & - & - \\
\hline Verano & - & - & 1 & 2 & 2 & 5 & 5 & 15 & 6 & 18 \\
\hline Verano + otoño & - & - & 2 & 5 & 1 & 2 & 2 & 6 & 6 & 18 \\
\hline Primavera + otoño & - & - & 3 & 7 & - & - & - & - & - & - \\
\hline Invierno + verano & - & - & - & - & - & - & - & - & 7 & 20 \\
\hline Tres estaciones & 12 & 26 & 5 & 12 & 12 & 30 & 7 & 20 & 4 & 11 \\
\hline Cuatro estaciones & 14 & 30 & 4 & 10 & 6 & 15 & 4 & 11 & 1 & 3 \\
\hline
\end{tabular}

Tabla 4. Distribución estacional de las distintas fenofases en número de especies (n) y porcentaje respecto al total de número de especies leñosas mediterráneas analizadas (\%) en el Jardín Botánico de la Universidad de Málaga. Crecimiento vegetativo (CV).Floración (FL. Fructificación (FR). Dispersión de diáporas (DS). Caída de hojas (CH).

Table 4. Seasonal distribution of the different phenofases in number of species ( $n$ ) and percentage with respect to the total number of mediterranean woody species analyzed (\%) in the Botanical Garden of the University of Malaga. Vegetative growth (CV). Flowering (FL). Fructification (FR). Dispersion of diapores (DS). Leaf fall (CH)

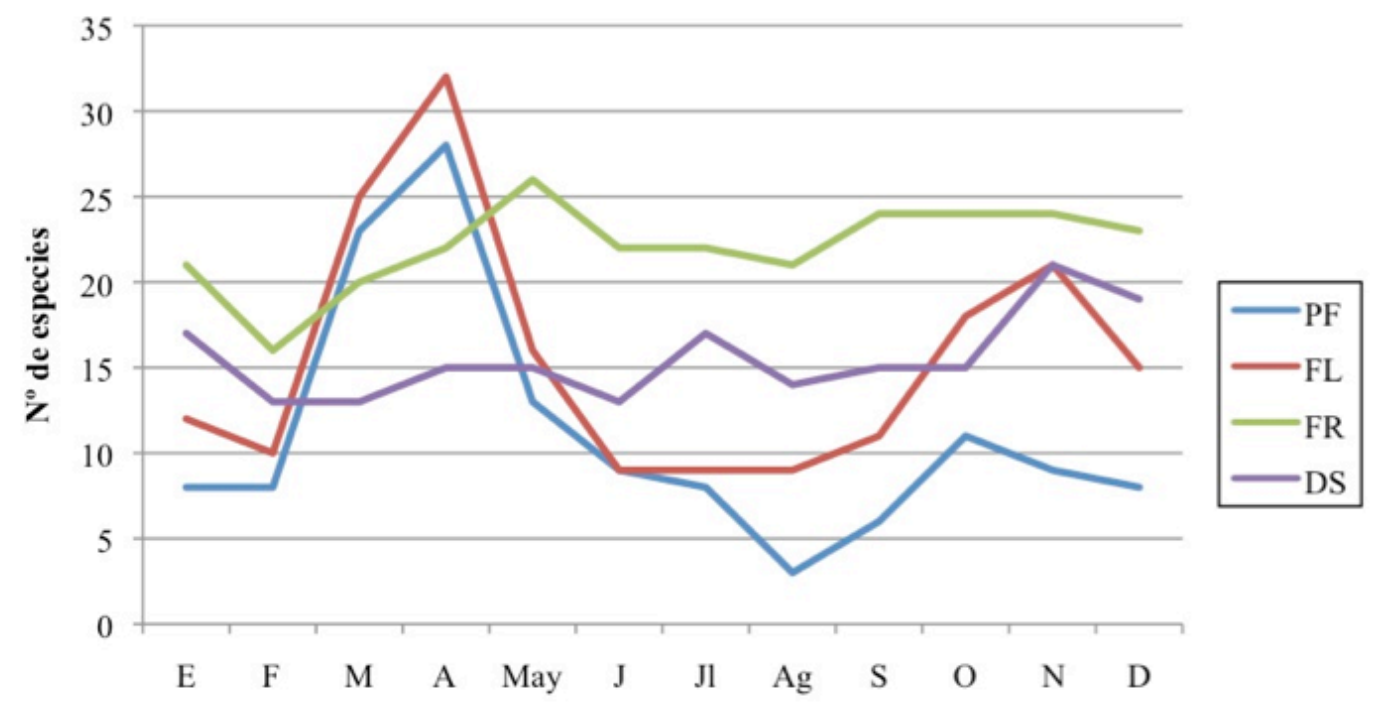

Meses del año

Figura 3. Número de especies leñosas mediterráneas que muestran fenofases reproductivas a lo largo de los meses del periodo estudiado (Julio 2014 - Junio 2015) en el Jardín Botánico de la Universidad de Málaga: formación de yemas florales (PF), floración (FL), fructificación (FR), dispersión (DS).

Figure 3. Number of Mediterranean woody species that show reproductive phenofases throughout the months of the period studied (July 2014 - June 2015) in the Botanical Garden of the University of Malaga: formation of floral buds (FP), flowering (FL), fructification (FR), dispersion (DS). 


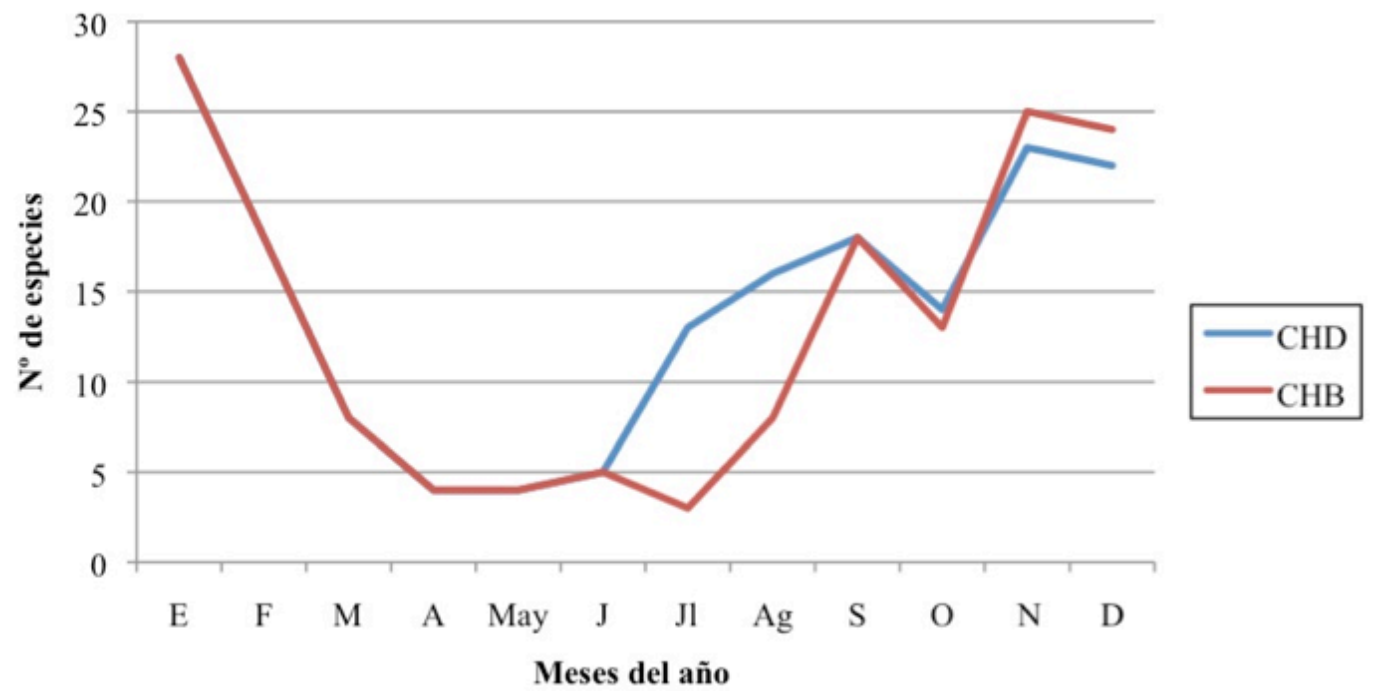

Figura 4. Número de especies leñosas mediterráneas que muestran fenofases de caída de hoja de dolicoblastos (CHD) y braquiblastos (CHB) a lo largo de los meses del periodo estudiado (Julio 2014 - Junio 2015) en el Jardín Botánico de la Universidad de Málaga.

Figure 4. Number of Mediterranean woody species that show phenofases of dolichoblasts (CHD) and brachyblasts (CHB) leaf fall throughout the months of the period studied (July 2014 - June 2015) in the Botanical Garden of the University of Malaga.

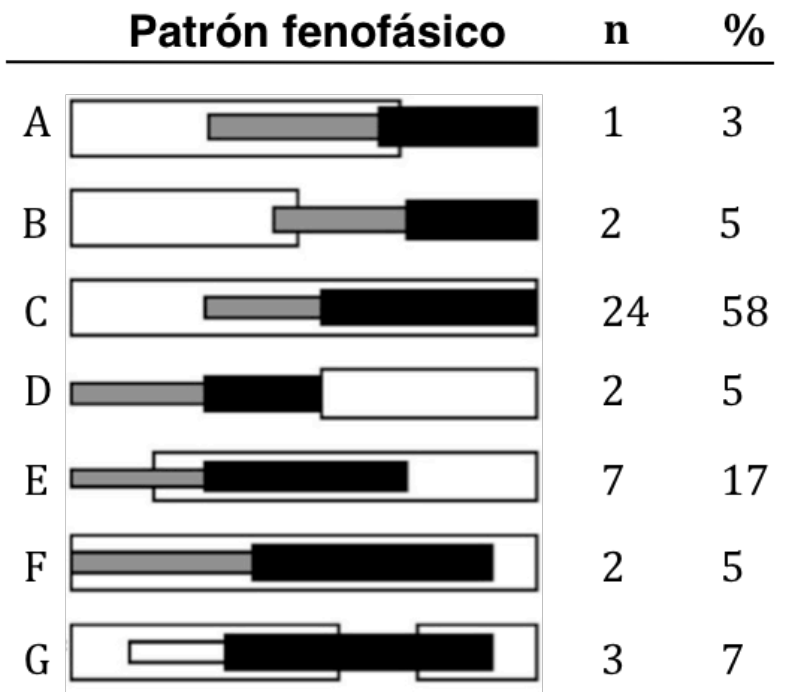

Figura 5. Patrones fenofásicos obtenidos por las especies leñosas mediterráneas del Jardín Botánico de la Universidad de Málaga. Crecimiento vegetativo (rectángulo blanco). Formación de yemas florales (rectángulo gris) . Floración (rectángulo negro). Número de especies (n). Porcentajes respecto al total de especies examinadas $(\%)$.

Figure 5. Phenophasic patterns obtained by the Mediterranean woody species in the Botanical Garden of the University of Malaga. Vegetative growth (white rectangle). Flowerbud formation (grey rectangle). Flowering (black rectangle). Number of species (n). Percentages with respect to the total of species examined (\%). fenológicas coinciden con cambios estacionales en las condiciones climáticas típicas del mediterráneo.

En el jardín estudiado existen 11 especies caducifolias de distribución mediterránea y tres especies leñosas áfilas (sin hojas ni filoclados). El resto de las especies leñosas mediterráneas son perennifolias pero presentan caída parcial de hojas. En general la fenofase de caída de hojas para la gran mayoría de las especies estudiadas ocurre durante el verano, sobre todo a finales, pero también hay una buena representatividad durante el otoño y principios de invierno, siendo muy escasas las especies que lo hacen durante primavera.

Se han determinado siete patrones fenofásicos distintos entre las especies leñosas mediterráneas cultivadas en el jardín. La gran mayoría presentan patrones fenofásicos de tipo superpuesto, en los que el crecimiento vegetativo y la floración están solapados en su ciclo anual, siendo minoritarios los patrones de tipo secuencial.

Los distintos comportamientos fenofásicos son consecuencia de su adaptación al medio, por lo que estos tipos de estudios dan información muy útil para estudiar cambios globales como consecuencia del cambio climático.

Los resultados fenológicos que se obtengan de los ejemplares cultivados en el jardín se pueden extrapolar a los que habitan en medios naturales. En este trabajo se demuestra que los comportamientos fenológicos son constantes 
para cada especie de angiosperma, estando conservados en su patromonio genético. Por ello las angiospermas leñosas mediterráneas de nuestro jardín constituyen un recurso vivo muy útil para realizar múltiples trabajos de investigación.

\section{Agradecimientos}

Este trabajo constituye uno de los resultados de los proyectos de innovación educativa que se han llevado a cabo durante los últimos años en la Universidad de Málaga: PIE07-079 y PIE08-071. Las autoras quisieran agradecer al Dr. Manuel Marí Beffa la iniciativa de este proyecto, al Dr. Alfredo Asensi Marfil la donación del catálogo inicial de especies del jardín, y a la Dra. Noelia Hidalgo Triana la ayuda para la identificación de los crecimientos vegetativos de dolicoblastos y braquiblastos de algunas especies en el jardín.

\section{Bibliografía}

Aljaro, M.E., \& Montenegro, G. (1981). Growth of dominant Chilean shrubs in the Andean Cordillera. Mountai Reserach and Development, 1, 287-291.

Azcón, J., \& Talón, M. (2008). Fundamentos de Fisiología Vegetal (2aed.) (pp. 617-638). Madrid: McGraw-Hill Interamericana.

Blanca, G., Cabezudo, B., Cueto, M., Morales, C., \& Salazar, C. (2011). Flora Vascular de Andalucía Oriental. Granada: Universidades de Granada, Almería, Jaén y Málaga.

Cabezudo, B., Navarro, T., Pérez-Latorre, A.V., Nieto, J.M., \& Orshan, G. (1992). Estudios fenomorfológicos en la vegetación del sur de España. I. Cistus L. Acta Botanica Malacitana, 17, 229-237.

Castroviejo, S. (Coord.). (1986). Flora Ibérica. Plantas Vasculares de la Península Ibérica e Islas Baleares. Madrid: Real Jardín Botánico, CSIC.

Floret, CH., Galán, M.J., Le Floc'h, E., Leprince, F., \& Romane, F. 1989. Description on plant annual cycles. France. In G. Orshan (Ed.), Plant pheno-morphological studies in Mediterranean type ecosistems (pp. 9-97). Dordrecht: Klumer Academic Publishers.

Hidalgo, M.I., \& Cabezudo, B. (1994). Fenología y volumen de floración del matorral de la Sierra de Mijas (Málaga, S. España). Acta Botanica Malacitana, 19, 123-136.

Hidalgo-Triana, N., Pérez Latorre, A.V. \& Hanse Thorne, J. (2017). Plant functional traits and groups in a Californian serpentine chaparral. Ecological Research, DOI 10.1007/s11284-017-1532-6.

Hoffmann, A.J. (1972). Morphology and histology of Trevoa trinervis (Rhamnaceae) a drought-deciduous shrub from the Chilean matorral. Flora,16, 527-538.

Hoffmann, A.J. 1981. Seasonal growth rhythm in Peumus boldus a deciduous tree of the Chilean mediterranean vegetation. Ecology of Plant, 2: 31-39.

Hoffmann, A.J., \& Hoffmann, A.E. (1976). Growth patterns and seasonal behaviour of the buds os Colliguaya odorifera, a shrub from the Chilean mediterranean vegetation. Canadian Journal of Botany, 54, 1774-
1787.

Hoffmann, A.J., \& Walker, M.J. (1980). Growth habits and phenology of drought- decidous species in an altitudinal gradient. Canadian Journal of Botany, 58, 1789-1796.

Jow, W.M., Bullock, S.H., \& Kummerow, J. (1980). Leaf turnover rates of Adenostoma fasciculatum (Rosaceae). American Journal of Botany, 67, 256-261.

Le Roux, A. , Perry, P., \& Kyriacou, X. (1989). Description on plant annual cycles. South Africa. In G. Orshan (Ed.), Plant pheno-morphological studies in Mediterranean type ecosistems (pp. 159-346). Dordrecht: Klumer Academic Publishers.

Médali, F. \& Quétzel, P. (1999). Biodiversity Hotspots in Mediterranean Basin: Setting Global Conservation Priorities. Conservation Biology, 13, 1510-1513.

Montenegro, G. (1987). Quantification of mediterranean plant phenology and growth. In: Tenhunen, J.D., Catarino, F.M., Lange, O.L., Oechel, W.C. (eds.) Planta Response to Stress. NATO ASI Series, Series G Ecological Sciencies, vol. 15. Berlin: Springer.

Montenegro, G., Aljaro, M.E., \& Kummerow, J. (1979). Growth dynamics of Chilean matorral shrubs. Botanical Gazette, 140, 114-119.

Montenegro, G., Avila, G., Aljaro, M.E., Osorio, R., \& Gomez, M. (1989). Description on plant annual cycles. Chile. In G. Orshan (Ed), Plant pheno-morphological studies in Mediterranean type ecosistems (pp. 347387). Dordrecht: Klumer Academic Publishers.

Navarro, T. \& Cabezudo, B. (1998). Estrategias fenomorfológicas en especies de un matorral mediterráneo (Andalucía, España). Acta Botanica Malacitana, 23, 133-148.

Navarro, T., Pascual, V. Cabezudo, B. \& Alados, C. (2009). Architecture and functional traits of semi-arid shrub species in Cabo de Gata Natural Park, SE Spain. Candollea, 64, 69-84.

Orshan, G. (1964). Seasonal dimorphism of desert and mediterranean chamaephytes and their significance as a factor in their water economy. In A.J. Rutter \& F.H. Whiehead (Eds.), Water in relation to plants (pp. 206-222). Oxford: Blackwell.

Orshan, G. (1982). Monocharacter growth-form types as a tool in an analytic-synthetic study of growth-forms in Mediterranen type ecosistems. A proposal for an interregional program. In Ecologia mediterranea. Définition et localisation des écosystèmes méditerranéens terrestres. Saint-Maximin 16-20 November 1981 (pp. 159-171).

Orshan, G. (1986). Plant form as describing vegetation and expressing adaptation to environment. Annals of Botany, 44, 7-38.

Orshan, G. (1989). Plant pheno-morphological studies in Mediterranean type ecosistems. Dordrecht : Klumer Academic Publishers.

Palacio, S. \& Montserrat-Martí, G. (2006). Comparison of the bud morphology and shoot growth dynamics of four species of Mediterranean subshrub growing along an actitudinal gradient. Botanical Journal of Linnean Society, 151, 527-539.

Pérez Latorre, A.V., Cabezudo, B., Nieto, J.M., \& Navarro, T. (1996). Caracterización fenológica y ecomorfológica 
de alcornocales andaluces (Málaga, España). Anales del Jardín Botánico de Madrid, 54, 554-560.

Pérez-Latorre, A.V., \& Cabezudo, B. (2002). Use of monocharacteristic growth forms and phenological phases to describe and differentiate plant communities in Mediterranean-type ecosystems. Plant Ecology, 161, 231-249.

Pérez-Latorre, A.V., \& Cabezudo, B. (2006). Phenomorphological and eco-morphological characters of Rhododendron lauroid forest in the Western Mediterranean (Iberian Peninsula, Spain). Plat Ecology, 187, 227-247.

Pérez-Latorre, A.V., Gavira, O., \& Cabezudo, B. (2010). Ecomorphology and phenomorphologyof Mediterranean heathlands (SW Iberian Peninsula). Phytocoenologia, 37, 239-268.

Pérez-Latorre, A.V., Gavira, O., \& Cabezudo, B. (2010). Phenomorphology and ecomorphological caracteres of Maytenus senegalensis L. shrublands in the Iberian Peninsula: A comparison with other Mediterranean plant communities. Flora, 205, 200-210.

Rutherford, M.C., \& Panagos, M.D, (1982). Seasonal woody plant shoot growth in Burkea africana - Ochna pulchra savanna. South African Journal of Botany, 1, 104-116.

Spano, D., Cesaraccio, C., Dulce, P., \& Snyder, R.L. (1999). Phenological stages of natural species and their use as climate indicators. International Journal of Biometeorology, 42, 124-133.

Stebbins, G.L. (1974). Flowering Plants. Evolution above the Species Level. London: Harvard University Press.

The Angiosperm Phylogeny Group. (2009). An update of the Angiosperm Phylogeny Group classification for the orders and families of flowering plants: APG III. Botanical Journal of the Linnean Society,161, 105121.

Valdés, B., Talavera, S., \& Galiano, E. (1987). Flora Vascular de Andalucía Occidental. Barcelona: Ketres.

Westman, W.E. (1981). Seasonal dimorphism of foliage in Californian Coastal sage scrub. Oecologia, 51, 385388. 